Genni Fragnelli*

\title{
Null controllability for a degenerate population model in divergence form via Carleman estimates
}

https://doi.org/10.1515/anona-2020-0034

Received December 29, 2018; accepted May 16, 2019.

Abstract: In this paper we consider a degenerate population equation in divergence form depending on time, on age and on space and we prove a related null controllability result via Carleman estimates.

Keywords: population equation, degenerate equation, Carleman estimates, observability inequalities MSC: 35K65, 92D25, 93B05, 93B07

\section{Introduction}

We consider the following population model in divergence form describing the dynamics of a single species:

$$
\begin{cases}\frac{\partial y}{\partial t}+\frac{\partial y}{\partial a}-\left(k(x) y_{x}\right)_{x}+\mu(t, a, x) y=f(t, a, x) \chi_{\omega} & \text { in } Q, \\ y(t, a, 1)=y(t, a, 0)=0 & \text { on } Q_{T, A}, \\ y(0, a, x)=y_{0}(a, x) & \text { in } Q_{A, 1}, \\ y(t, 0, x)=\int_{0}^{A} \beta(a, x) y(t, a, x) d a & \text { in } Q_{T, 1} .\end{cases}
$$

Here $Q:=(0, T) \times(0, A) \times(0,1), Q_{T, A}:=(0, T) \times(0, A), Q_{A, 1}:=(0, A) \times(0,1)$ and $Q_{T, 1}:=(0, T) \times(0,1)$. Moreover, $y(t, a, x)$ is the distribution of certain individuals at location $x \in(0,1)$, at time $t \in(0, T)$, where $T$ is fixed, and of age $a \in(0, A) . A$ is the maximal age of life, while $\beta$ and $\mu$ are the natural fertility and the natural death rates, respectively. Thus, the formula $\int_{0}^{A} \beta y d a$ denotes the distribution of newborn individuals at time $t$ and location $x$. In the model $\chi_{\omega}$ is the characteristic function of the control region $\omega \subset(0,1)$; the function $k$ is the dispersion coefficient and we assume that it depends on the space variable $x$ and degenerates at the boundary of the state space. We say that the function $k$ is

Definition 1.1. Weakly degenerate (WD) if $k \in W^{1,1}([0,1])$,

$$
k>0 \text { in }(0,1) \text { and } k(0)=k(1)=0 \text {, }
$$

and there exist $M_{1}, M_{2} \in(0,1)$ such that $x k^{\prime}(x) \leq M_{1} k(x)$ and $(x-1) k^{\prime}(x) \leq M_{2} k(x)$ for a.e. $x \in[0,1]$.

or

Definition 1.2. Strongly degenerate (SD) if $k \in W^{1, \infty}([0,1])$,

$$
k>0 \text { in }(0,1) \text { and } k(0)=k(1)=0 \text {, }
$$

and there exist $M_{1}, M_{2} \in[1,2)$ such that $x k^{\prime}(x) \leq M_{1} k(x)$ and $(x-1) k^{\prime}(x) \leq M_{2} k(x)$ for a.e. $x \in[0,1]$.

*Corresponding Author: Genni Fragnelli, Dipartimento di Matematica, Università di Bari "Aldo Moro", Via E. Orabona 4, 70125 Bari, Italy, E-mail: genni.fragnelli@uniba.it 
For example, as $k$ one can consider $k(x)=x^{\alpha}(1-x)^{\beta}, \alpha, \beta>0$. Clearly, we say that $k$ is weakly or strongly degenerate only at 0 if Definition 1.1 is satisfied only at 0 , i.e. $k \in W^{1,1}([0,1]), k>0$ in $(0,1], k(0)=0$ and, there exists $M_{1} \in(0,1)$ or $M_{1} \in[1,2)$ such that $x k^{\prime}(x) \leq M_{1} k(x)$ for a.e. $x \in[0,1]$. Analogously at 1 .

In the last centuries, population models have been widely investigated by many authors from many points of view (see, for example, [5], [9], [14], [20]). From the general theory for the Lotka-McKendrick system, it is known that the asymptotic behavior of the solution depends on the so called net reproduction rate $R_{0}$ : if $R_{0}>1$, the solution is exponentially growing; if $R_{0}<1$, the solution is exponentially decaying; if $R_{0}=1$, the solution tends to the steady state solution. Clearly, if $R_{0}>1$ and the system represents the distribution of a damaging insect population or of a pest population, it is very worrying. For example, in $2017 \mathrm{~B}$. Zhong, C. Lv, W. Qin show that the net reproduction rate for the Tirathaba rufivena (which causes a lot of damages for the crop, for example, of fruits and flowers) depends on the temperature: it is 10.40 if the temperature is $28^{\circ} \mathrm{C}$ and it is 4.13 is the temperature is $20^{\circ} \mathrm{C}$ (see, for example, [25]); in $2011 \mathrm{~S}$. S. Win, R. Muhamad, Z. A. M. Ahmad, N. A. Adam show that the net reproduction rate for the Nilaparvata lugens (which caused a lot of damages for the rice crop throughout South and South-East Asia since the early 1970's) was about 10 (see, for example, [24]). For this reason, recently great attention is given to null controllability. For example in [21], where (1.1) models an insect growth, the control corresponds to a removal of individuals by using pesticides.

There are a lot of papers that deal with null controllability for (1.1) when the dispersion coefficient $k$ is $a$ constant or a strictly positive function (see, for example, [3]). If $y$ is independent of $a$ and $k$ degenerates at the boundary or at an interior point of the domain we refer, for example, to [2], [15] and to [17], [18], [19] if $\mu$ is singular at the same point of $k$. To our best knowledge, [1] is the first paper where $y$ depends on $t, a$ and $x$ and the dispersion coefficient $k$ can degenerate. In particular, the authors assume that $k$ degenerates at the boundary (for example $k(x)=x^{\alpha}$, being $x \in(0,1)$ and $\alpha>0$ ). Using Carleman estimates for the adjoint problem, the authors prove null controllability for (1.1) under the condition $T \geq A$. However, this assumption is not realistic when $A$ is too large. To overcome this problem in [10], the authors used Carleman estimates and a fixed point method via the Leray - Schauder Theorem. However, in [10] the authors consider a dispersion coefficient that can degenerate only at a point of the boundary and they use the fixed point technique in which the birth rate $\beta$ must be in $C^{2}(Q)$ specially in the proof of [10, Proposition 4.2]. In the recent paper [13], we studied null controllability for (1.1) in non divergence form and with a diffusion coefficient degenerating at a one point of the boundary domain or in an interior point. Observe that, in the case of a boundary degeneracy, we cannot derive the null controllability for (1.1) by the one of the problem in non divergence form or vice versa, see [7]. For this reason here we study the null controllability for (1.1) assuming that $k$ degenerates at the boundary of the domain and $T<A$ completing [1]. We underline that here, contrary to [10] and [13], we assume also that $k$ can degenerate at both points of the boundary domain (see Theorem 4.8) and $\beta$ is only a continuous function. On the other hand, while in [10] the authors used Carleman estimates, a generalization of the Leray - Schauder fixed point Theorem and the multi-valued theory, here we use only Carleman estimates, some results of [13] and a technique based on cut off functions, making the proof slimmer and easier to read. Moreover, the technique that we use to prove Theorem $4.8 \mathrm{can}$ be applied also to the problem in non divergence form considered in [13], generalizing [13, Theorem 4.8]. Finally, in the proof of the last theorem we make precise a calculation of [13, Theorem 4.8] which was not accurate. Observe that in this paper, as in [13], we do not consider the positivity of the solution, even if it is clearly interesting. This problem is related to the minimum time issue, i.e. given $T$ cannot be arbitrarily small, but this study is still a work in progress, see [23] for related results in non degenerate cases.

A final comment on the notation: by $c$ or $C$ we shall denote universal strictly positive constants, which are allowed to vary from line to line.

\section{Well posedness results}

On the rates $\mu$ and $\beta$ we assume: 
Hypothesis 2.1. The functions $\mu$ and $\beta$ are such that

$$
\begin{aligned}
& \text { - } \beta \in C\left(\bar{Q}_{A, 1}\right) \text { and } \beta \geq 0 \text { in } Q_{A, 1}, \\
& \text { - } \mu \in C(\bar{Q}) \text { and } \mu \geq 0 \text { in } Q .
\end{aligned}
$$

To prove well posedness of (1.1), we introduce, as in [2], the following Hilbert spaces

$$
\begin{array}{r}
H_{k}^{1}:=\left\{u \in L^{2}(0,1) \mid u \text { absolutely continuous in }[0,1],\right. \\
\left.\sqrt{k} u_{x} \in L^{2}(0,1) \text { and } u(1)=u(0)=0\right\}
\end{array}
$$

and

$$
H_{k}^{2}:=\left\{u \in H_{k}^{1}(0,1) \mid k u_{x} \in H^{1}(0,1)\right\} .
$$

We have, as in [2] or [16], that the operator

$$
\mathcal{A}_{0} u:=\left(k u_{x}\right)_{x}, \quad D\left(\mathcal{A}_{0}\right):=\mathcal{H}_{k}^{2}(0,1)
$$

is self-adjoint, nonpositive and generates a strongly continuous semigroup on the space $L^{2}(0,1)$.

Now, setting $\mathcal{A}_{a} u:=\frac{\partial u}{\partial a}$, we have that

$$
\mathcal{A} u:=\mathcal{A}_{a} u-\mathcal{A}_{0} u,
$$

for

$$
u \in D(\mathcal{A})=\left\{u \in L^{2}\left(0, A ; D\left(\mathcal{A}_{0}\right)\right): \frac{\partial u}{\partial a} \in L^{2}\left(0, A ; H_{k}^{1}(0,1)\right), u(0, x)=\int_{0}^{A} \beta(a, x) u(a, x) d a\right\},
$$

generates a strongly continuous semigroup on $L^{2}\left(Q_{A, 1}\right):=L^{2}\left(0, A ; L^{2}(0,1)\right)$ (see also [4]). Moreover, the operator $B(t)$ defined as

$$
B(t) u:=\mu(t, a, x) u,
$$

for $u \in D(\mathcal{A})$, can be seen as a bounded perturbation of $\mathcal{A}$ (see, for example, [2]); thus also $(\mathcal{A}+B(t), D(\mathcal{A})$ ) generates a strongly continuous semigroup.

Setting $L^{2}(Q):=L^{2}\left(0, T ; L^{2}\left(Q_{A, 1}\right)\right)$, the following well posedness result holds:

Theorem 2.1. Assume that $k$ is weakly or strongly degenerate at 0 and/or at 1 . For all $f \in L^{2}(Q)$ and $y_{0} \in$ $L^{2}\left(Q_{A, 1}\right)$, the system (1.1) admits a unique solution

$$
\left.y \in \mathcal{U}:=C\left([0, T] ; L^{2}\left(Q_{A, 1}\right)\right)\right) \cap L^{2}\left(0, T ; H^{1}\left(0, A ; H_{k}^{1}(0,1)\right)\right)
$$

and

$$
\sup _{t \in[0, T]}\|y(t)\|_{L^{2}\left(Q_{A, 1}\right)}^{2}+\int_{0}^{T} \int_{0}^{A}\left\|\sqrt{k} y_{x}\right\|_{L^{2}(0,1)}^{2} d a d t \leq C\left\|y_{0}\right\|_{L^{2}\left(Q_{A, 1}\right)}^{2}+C\|f\|_{L^{2}(Q)}^{2},
$$

where $C$ is a positive constant independent of $k, y_{0}$ and $f$.

In addition, if $\equiv \equiv$, then $y \in C^{1}\left([0, T] ; L^{2}\left(Q_{A, 1}\right)\right)$.

For the existence of the solution and the regularity of it we refer, for example, to [11] and [22]. On the other hand, we postpone the proof of (2.2) to the Appendix.

\section{Carleman estimates}

From the general theory, it is known that null controllability for a linear parabolic system is, roughly speaking, equivalent to the observability for the associated homogeneous adjoint problem (see, for example, [12]). Thus, 
the key point is to prove such an inequality. A usual strategy in showing the observability inequality is to prove that certain global Carleman estimates hold true for the adjoint operator. Hence, this section is devoted to obtain global Carleman estimates for the operator which is the adjoint of the given one in both the weakly and the strongly degenerate cases. In particular, we consider the following adjoint system associated to (1.1):

$$
\begin{cases}\frac{\partial z}{\partial t}+\frac{\partial z}{\partial a}+\left(k(x) z_{x}\right)_{x}-\mu(t, a, x) z=f, & (t, a, x) \in Q \\ z(t, a, 0)=z(t, a, 1)=0, & (t, a) \in Q_{T, A} \\ z(t, A, x)=0, & (t, x) \in Q_{T, 1}\end{cases}
$$

\subsubsection{Carleman inequalities when the degeneracy is at 0 .}

In this subsection we will consider the case when $k(0)=0$ and we assume that $\mu$ satisfies (2.1). On the other hand, on $k$ we make additional assumptions:

Hypothesis 3.1. The function $k \in C^{0}[0,1] \cap C^{1}(0,1]$ is such that $k(0)=0, k>0$ on $(0,1]$ and there exists $M_{1} \in(0,2)$ such that $x k^{\prime}(x) \leq M_{1} k(x)$ for all $x \in[0,1]$. Moreover, if $M_{1} \geq 1$ one has to require that there exists $\theta \in\left(0, M_{1}\right]$, such that the function $x \mapsto \frac{k(x)}{x^{\theta}}$ is nondecreasing near 0 .

We remark that the assumption $x a^{\prime} \leq M_{1} a$, with $M_{1}<2$, is essential in all our results and it is the same made, for example, in [2]. It implies that $\frac{1}{\sqrt{a}} \in L^{1}(0,1)$ and, in particular, if $K<1$, then $\frac{1}{a} \in L^{1}(0,1)$. Thus, the case $M_{1} \geq 2$ is excluded. Summing up, we will confine our analysis to the case of $\frac{1}{\sqrt{a}} \in L^{1}(0,1)$. This is, however, the interesting case from the viewpoint of null controllability. In fact, if $\frac{1}{\sqrt{a}} \notin L^{1}(0,1)$ and $y$ is independent of $a$, then (1.1) fails to be null controllable on the whole interval [0, 1], and regional null controllability is the only property that can be expected, see [8].

Moreover, the assumption “ $\exists \theta$ such that the function $x \rightarrow \frac{k(x)}{x^{\theta}}$ is nondecreasing near 0 ” is just technical and it is equivalent to the following one: " $\exists \theta$ such that $\theta a \leq x a^{\prime}$ near 0 ". Clearly, the prototype is $k(x)=$ $x^{\alpha}, \alpha \in(0,2)$.

Now, let us introduce the weight function

$$
\varphi(t, a, x):=\Theta(t, a)\left(p(x)-2\|p\|_{L^{\infty}(0,1)}\right)
$$

where $\Theta$ is as in (4.7) and $p(x):=\int_{0}^{x} \frac{y}{k(y)} d y$. Observe that $\varphi(t, a, x)<0$ for all $(t, x) \in Q$ and $\varphi(t, a, x) \rightarrow$ $-\infty$ as $t \rightarrow 0^{+}, T^{-}$or $a \rightarrow 0^{+}$. The following estimate holds:

Theorem 3.1. Assume that Hypothesis 3.1 is satisfied. Then, there exist two strictly positive constants $C$ and $s_{0}$ such that every solution $v$ of (3.1) in

$$
\vee:=L^{2}\left(Q_{T, A} ; H_{k}^{2}(0,1)\right) \cap H^{1}\left(0, T ; H^{1}\left(0, A ; H_{k}^{1}(0,1)\right)\right)
$$

satisfies, for all $s \geq s_{0}$,

$$
\int_{Q}\left(s \Theta k v_{x}^{2}+s^{3} \Theta^{3} \frac{x^{2}}{k} v^{2}\right) e^{2 s \varphi} d x d a d t \leq C \int_{Q} f^{2} e^{2 s \varphi} d x d a d t+s C \int_{0}^{T} \int_{0}^{A} \Theta(t, a)\left[k v_{x}^{2} e^{2 s \varphi}\right](t, a, 1) d a d t .
$$

Clearly the previous Carleman estimate holds for every function $v$ that satisfies (3.1) in $(0, T) \times(0, A) \times(B, C)$ as long as $(0,1)$ is substituted by $(B, C)$ and $k$ satisfies Hypothesis 3.1 in $(B, C)$. 


\subsubsection{Proof of Theorem 3.1}

As a first step assume that $\mu \equiv 0$.

In order to prove Theorem 3.1, we define, for $s>0$, the function

$$
w(t, a, x):=e^{s \varphi(t, a, x)} v(t, a, x)
$$

where $v$ is the solution of (3.1) in $\mathcal{V}$; observe that, since $v \in \mathcal{V}, w \in \mathcal{V}$. Clearly, one has that $w$ satisfies

$$
\begin{cases}\left(e^{-s \varphi} w\right)_{t}+\left(e^{-s \varphi} w\right)_{a}+\left(k\left(e^{-s \varphi} w\right)_{x}\right)_{x}=f(t, a, x), & (t, x) \in Q, \\ w(0, a, x)=w(T, a, x)=0, & (a, x) \in Q_{A, 1}, \\ w(t, A, x)=w(t, 0, x)=0, & (t, x) \in Q_{T, 1}, \\ w(t, a, 0)=w(t, a, 1)=0, & (t, a) \in Q_{T, A} .\end{cases}
$$

Defining $L w:=w_{t}+w_{a}+\left(k w_{x}\right)_{x}$ and $L_{s} w:=e^{s \varphi} L\left(e^{-s \varphi} w\right)$, the equation of (3.3) can be recast as follows

$$
L_{s} w=L_{s}^{+} w+L_{s}^{-} w=e^{s \varphi} f,
$$

where

$$
\left\{\begin{array}{l}
L_{s}^{+} w:=\left(k w_{x}\right)_{x}-s\left(\varphi_{t}+\varphi_{a}\right) w+s^{2} k \varphi_{x}^{2} w, \\
L_{s}^{-} w:=w_{t}+w_{a}-2 s k \varphi_{x} w_{x}-s\left(k \varphi_{x}\right)_{x} w .
\end{array}\right.
$$

As usual, we compute the inner product $\left\langle L_{S}^{+} w, L_{s}^{-} w>_{L^{2}(Q)}\right.$ whose first expression is given in the following lemma

Lemma 3.1. Assume Hypothesis 3.1. The following identity holds

$$
\begin{aligned}
& \left\langle L_{s}^{+} w, L_{s}^{-} w\right\rangle_{L^{2}(Q)}=\frac{s}{2} \int_{Q}\left(\varphi_{t t}+\varphi_{a a}\right) w^{2} d x d a d t+s \int_{Q} k(x)\left(k(x) \varphi_{x}\right)_{x x} w w_{x} d x d a d t \\
& -2 s^{2} \int_{Q} k \varphi_{x} \varphi_{t x} w^{2} d x d a d t-2 s^{2} \int_{Q} k \varphi_{x} \varphi_{x a} w^{2} d x d a d t \\
& +s \int_{Q}\left(2 k^{2} \varphi_{x x}+k k^{\prime} \varphi_{x}\right) w_{x}^{2} d x d a d t+s^{3} \int_{Q}\left(2 k \varphi_{x x}+k^{\prime} \varphi_{x}\right) k \varphi_{x}^{2} w^{2} d x d a d t \\
& +s \int_{Q} \varphi_{a t} w^{2} d x d a d t \\
& \left\{\begin{array}{l}
\int_{Q_{T, A}}\left[k w_{x} w_{t}\right]_{0}^{1} d a d t+\int_{Q_{T, A}}\left[k w_{x} w_{a}\right]_{0}^{1} d a d t-\frac{s}{2} \int_{Q_{A, 1}}\left[\varphi_{a} w^{2}\right]_{0}^{T} d x d a \\
+\int_{Q_{T, A}}\left[-s \varphi_{x}\left(k(x) w_{x}\right)^{2}+s^{2} k(x) \varphi_{t} \varphi_{x} w^{2}-s^{3} k^{2} \varphi_{x}^{3} w^{2}\right]_{0}^{1} d a d t \\
+\int_{Q_{T, A}}\left[-s k(x)\left(k(x) \varphi_{x}\right)_{x} w w_{x}\right]_{0}^{1} d a d t+s^{2} \int_{Q_{T, A}}\left[k \varphi_{x} \varphi_{a} w^{2}\right]_{0}^{1} d a d t \\
-\frac{1}{2} \int_{Q_{T, 1}}\left[k w_{x}^{2}\right]_{0}^{A} d x d t+\frac{1}{2} \int_{Q_{T, 1}}\left[\left(s^{2} k \varphi_{x}^{2}-s\left(\varphi_{t}+\varphi_{a}\right)\right) w^{2}\right]_{0}^{A} d x d t
\end{array}\right.
\end{aligned}
$$

Proof. It results, integrating by parts,

$$
<L_{s}^{+} w, L_{s}^{-} w>_{L^{2}(Q)}=I_{1}+I_{2}+I_{3}+I_{4},
$$


where

$$
\begin{gathered}
I_{1}=\int_{Q}\left(k w_{x}\right)_{x}\left(w_{t}-2 s k \varphi_{x} w_{x}-s\left(k \varphi_{x}\right)_{x} w\right) d x d a d t, \\
I_{2}=\int_{Q}\left(-s \varphi_{t} w+s^{2} k \varphi_{x}^{2} w\right)\left(w_{t}-2 s k \varphi_{x} w_{x}-s\left(k \varphi_{x}\right)_{x} w\right) d x d a d t, \\
I_{3}=\int_{Q}\left(\left(k w_{x}\right)_{x}-s\left(\varphi_{t}+\varphi_{a}\right) w+s^{2} k \varphi_{x}^{2} w\right) w_{a} d x d a d t
\end{gathered}
$$

and

$$
I_{4}=-s \int_{Q} \varphi_{a} w\left(w_{t}-2 s k \varphi_{x} w_{x}-s\left(k \varphi_{x}\right)_{x} w\right) d x d a d t .
$$

By [2, Lemma 3.1], we get

$$
\begin{aligned}
I_{1}+I_{2} & :=\frac{s}{2} \int_{Q} \varphi_{t t} w^{2} d x d a d t+s \int_{Q} k(x)\left(k(x) \varphi_{x}\right)_{x x} w w_{x} d x d a d t \\
& -2 s^{2} \int_{Q} k(x) \varphi_{x} \varphi_{t x} w^{2} d x d a d t+s \int_{Q}\left(2 k^{2} \varphi_{x x}+k(x) k^{\prime} \varphi_{x}\right) w_{x}^{2} d x d a d t \\
& +s^{3} \int_{Q}\left(2 k(x) \varphi_{x x}+k^{\prime} \varphi_{x}\right) k(x) \varphi_{x}^{2} w^{2} d x d a d t+\int_{Q_{T, A}}\left[k(x) w_{x} w_{t}\right]_{x=0}^{x=1} d a d t \\
& +\int_{Q_{T, A}}\left[-s \varphi_{x}\left(k(x) w_{x}\right)^{2}+s^{2} k(x) \varphi_{t} \varphi_{x} w^{2}-s^{3} k^{2} \varphi_{x}^{3} w^{2}\right]_{x=0}^{x=1} d a d t \\
& +\int_{Q_{T, A}}\left[-s k(x)\left(k(x) \varphi_{x}\right)_{x} w w_{x}\right]_{x=0}^{x=1} d a d t .
\end{aligned}
$$

Next, we compute $I_{3}$ and $I_{4}$. Integrating by parts, we have

$$
\begin{aligned}
I_{3} & =-\frac{1}{2} \int_{0}^{T} \int_{0}^{1}\left[k w_{x}^{2}\right]_{0}^{A} d x d t+\int_{0}^{T} \int_{0}^{A}\left[k w_{x} w_{a}\right]_{0}^{1} d a d t \\
& +\frac{1}{2} \int_{0}^{T} \int_{0}^{1}\left[\left(s^{2} k \varphi_{x}^{2}-s\left(\varphi_{t}+\varphi_{a}\right)\right) w^{2}\right]_{0}^{A} d x d t \\
& +\frac{s}{2} \int_{Q} \varphi_{a a} w^{2} d x d a d t+\frac{s}{2} \int_{Q} \varphi_{t a} w^{2} d x d a d t-s^{2} \int_{Q} k \varphi_{x} \varphi_{x a} w^{2} d x d a d t .
\end{aligned}
$$

On the other hand

$$
\begin{aligned}
I_{4} & =\frac{s}{2} \int_{Q} \varphi_{a t} w^{2} d x d a d t-s^{2} \int_{Q} k \varphi_{x} \varphi_{a x} w^{2} d x d a d t \\
& -\frac{s}{2} \int_{0}^{A} \int_{0}^{1}\left[\varphi_{a} w^{2}\right]_{0}^{T} d x d a+s^{2} \int_{0}^{T} \int_{0}^{A}\left[k \varphi_{x} \varphi_{a} w^{2}\right]_{0}^{1} d a d t .
\end{aligned}
$$

Adding (3.5) - (3.7), (3.4) follows immediately.

As a consequence of the definition of $\varphi$, one has the next estimate:

Lemma 3.2. Assume Hypothesis 3.1. There exist two strictly positive constants $C$ and $s_{0}$ such that, for all $s \geq s_{0}$, all solutions $w$ of (3.3) satisfy the following estimate

$$
s C \int_{Q} \Theta k w_{x}^{2} d x d a d t+s^{3} C \int_{Q} \Theta^{3} \frac{x^{2}}{k} w^{2} d x d a d t \leq\{D . T .\} .
$$


Proof. The distributed terms of $\left\langle L_{S}^{+} w, L_{S}^{-} w\right\rangle_{L^{2}(Q)}$ take the form

$$
\begin{aligned}
\{D . T .\} & =\frac{s}{2} \int_{Q}\left(\Theta_{t t}+\Theta_{a a}\right)\left(p-2\|p\|_{L^{\infty}(0,1)}\right) w^{2} d x d a d t \\
& -2 s^{2} \int_{Q} \Theta_{t} \frac{x^{2}}{k} w^{2} d x d a d t-2 s^{2} \int_{Q} \Theta \Theta_{a} \frac{x^{2}}{k} w^{2} d x d a d t \\
& +s \int_{Q} \Theta\left(2 k-k^{\prime} x\right) w_{x}^{2} d x d a d t+s^{3} \int_{Q} \Theta^{3}\left(2 k-k^{\prime} x\right) \frac{x^{2}}{k^{2}} w^{2} d x d a d t \\
& +s \int_{Q} \Theta_{t a}\left(p-2\|p\|_{L^{\infty}(0,1)}\right) w^{2} d x d a d t .
\end{aligned}
$$

Now, observe that there exists $c>0$ such that

$$
\begin{aligned}
& \Theta^{\mu} \leq c \Theta^{v} \text { if } 0<\mu<v \\
& \left|\Theta \Theta_{t}\right| \leq c \Theta^{3},\left|\Theta \Theta_{a}\right| \leq c \Theta^{3}, \\
& \left|\Theta_{a a}\right| \leq c \Theta^{\frac{3}{2}},\left|\Theta_{t t}\right| \leq c \Theta^{\frac{3}{2}} \text { and }\left|\Theta_{t a}\right| \leq c \Theta^{\frac{3}{2}} .
\end{aligned}
$$

Hence, proceeding as in the proof of [2, Lemma 3.5], one can deduce

$$
\begin{aligned}
(3.8) \geq & \frac{s}{2} \int_{Q}\left(\Theta_{t t}+\Theta_{a a}\right)\left(p-2\|p\|_{L^{\infty}(0,1)}\right) w^{2} d x d a d t \\
& -s^{3} \frac{C}{4} \int_{Q} \Theta^{3} \frac{x^{2}}{k} w^{2} d x d a d t-s^{3} \frac{C}{4} \int_{Q} \Theta^{3} \frac{x^{2}}{k} w^{2} d x d a d t \\
& +C s \int_{Q} \Theta k w_{x}^{2} d x d a d t+s^{3} C \int_{Q} \Theta^{3} \frac{x^{2}}{k} w^{2} d x d a d t \\
& +s \int_{Q} \Theta_{t a}\left(p-2\|p\|_{L^{\infty}(0,1)}\right) w^{2} d x d a d t .
\end{aligned}
$$

Now, it results

$$
s\left|\int_{Q} \Theta_{t t}\left(p-2\|p\|_{L^{\infty}(0,1)}\right)\right| \leq \frac{C}{2} s\left|\int_{Q} \Theta^{3 / 2} b(x) w^{2} d x d a d t\right|+s \frac{C}{2}\left|\int_{Q} \Theta^{3 / 2} w^{2} d x d a d t\right|,
$$

where $b(x)=\int_{0}^{x} \frac{y}{k(y)} d y$. As in [2, Lemma 3.5], one can estimate the last two terms in the following way

$$
\frac{s}{2} \int_{Q} \Theta^{3 / 2} b(x) w^{2} d x d a d t \leq \frac{C}{16} s^{3} \int_{Q} \Theta^{3} \frac{x^{2}}{k(x)} w^{2} d x d a d t
$$

for $s$ large enough and

$$
\frac{s}{2}\left|\int_{Q} \Theta^{3 / 2} w^{2} d x d a d t\right| \leq \frac{C}{4} s \int_{Q} \Theta k(x) w_{x}^{2} d x d a d t+\frac{C}{16} s^{3} \int_{Q} \Theta^{3} \frac{x^{2}}{k(x)} w^{2} d x d a d t .
$$

Hence

$$
s\left|\int_{Q} \Theta_{t t} \psi(x) w^{2} d x d a d t\right| \leq \frac{C}{4} s \int_{0}^{1} \Theta k(x) w_{x}^{2} d x d a d t+\frac{C}{8} s^{3} \int_{Q} \Theta^{3} \frac{x^{2}}{k(x)} w^{2} d x d a d t .
$$

The same estimate holds also for

$$
\int_{Q} \Theta_{a a}\left(p-2\|p\|_{L^{\infty}(0,1)}\right) d x d a d t \quad \text { and } \quad \int_{Q} \Theta_{t a}\left(p-2\|p\|_{L^{\infty}(0,1)}\right) d x d a d t .
$$

Using the above estimates in (3.10) the thesis follows immediately for $s_{0}$ large enough. 
The next lemma holds.

Lemma 3.3. Assume Hypothesis 3.1. The boundary terms in (3.4) become

$$
\{B . T .\}=-\int_{Q_{T, A}}\left[s \Theta x k w_{x}^{2}\right]_{0}^{1} d a d t .
$$

Proof. Using the definition of $\varphi$, [2, Lemma 3.6], the boundary conditions of $w$ and proceeding as in [13, Lemma 3.2], the boundary terms of $\left\langle L_{s}^{+} w, L_{s}^{-} w\right\rangle_{L^{2}(Q)}$ become

$$
\begin{aligned}
\{B . T .\} & =\int_{Q_{T, A}}\left[k w_{x} w_{a}\right]_{0}^{1} d a d t-\frac{s}{2} \int_{Q_{A, 1}}\left[\varphi_{a} w^{2}\right]_{0}^{T} d x d a-\int_{Q_{T, A}}\left[s \Theta x k w_{X}^{2}\right]_{0}^{1} d a d t \\
& +s^{2} \int_{Q_{T, A}}\left[k \varphi_{x} \varphi_{a} w^{2}\right]_{0}^{1} d a d t-\frac{1}{2} \int_{Q_{T, 1}}\left[k w_{x}^{2}\right]_{0}^{A} d x d t \\
& +\frac{1}{2} \int_{Q_{T, 1}}\left[\left(s^{2} k \varphi_{x}^{2}-s\left(\varphi_{t}+\varphi_{a}\right)\right) w^{2}\right]_{0}^{A} d x d t \\
& =-\int_{Q_{T, A}}\left[s \Theta x k w_{X}^{2}\right]_{0}^{1} d a d t .
\end{aligned}
$$

As a consequence of Lemmas 3.3 and 3.2, we have

Proposition 3.1. Assume Hypothesis 3.1. There exist two strictly positive constants $C$ and $s_{0}$ such that, for all $s \geq s_{0}$, all solutions $w$ of (3.3) in $\mathcal{V}$ satisfy

$$
\begin{aligned}
& s C \int_{Q} \Theta k w_{x}^{2} d x d a d t+s^{3} C \int_{Q} \Theta^{3} \frac{x^{2}}{k} w^{2} d x d a d t \\
& \leq C\left(\int_{Q} f^{2} e^{2 s \varphi} d x d a d t+\int_{Q_{T, A}}\left[s \Theta k w_{x}^{2}\right](t, a, 1) d a d t\right) .
\end{aligned}
$$

Recalling the definition of $w$, we have $v=e^{-s \varphi} w$ and $v_{x}=\left(w_{x}-s \varphi_{x} w\right) e^{-s \varphi}$. Thus, Theorem 3.1 follows immediately by Proposition 3.1 when $\mu \equiv 0$.

Now, we assume that $\mu \not \equiv 0$.

To complete the proof of Theorem 3.1 we consider the function $\bar{f}=f+\mu v$. Hence, there are two strictly positive constants $C$ and $s_{0}$ such that, for all $s \geq s_{0}$, the following inequality holds

$$
\begin{aligned}
\int_{Q}\left(s \Theta k v_{x}^{2}+s^{3} \Theta^{3} \frac{x^{2}}{k} v^{2}\right) e^{2 s \varphi} d x d a d t & \leq C \int_{Q} \bar{f}^{2} e^{2 s \varphi} d x d a d t \\
& +s C \int_{0}^{T} \int_{0}^{A} \Theta(t, a)\left[k v_{x}^{2} e^{2 s \varphi}\right](t, a, 1) d a d t .
\end{aligned}
$$

On the other hand, we have

$$
\int_{Q}|\bar{f}|^{2} e^{2 s \varphi} d x d a d t \leq 2\left(\int_{Q}|f|^{2} e^{2 s \varphi} d x d a d t+\int_{Q}|\mu|^{2}|v|^{2} e^{2 s \varphi} d x d a d t\right) .
$$


Now, if $M_{1}<1$, applying the Hardy-Poincaré proved in [2, Proposition 2.1] to the function $v:=e^{s \varphi} v$, we obtain

$$
\begin{aligned}
\int_{Q}|\mu|^{2}|v|^{2} e^{2 s \varphi} d x d a d t & \leq\|\mu\|_{\infty}^{2} \int_{Q} v^{2} d x d a d t \leq C \int_{Q} v^{2} \frac{k(x)}{x^{2}} d x d a d t \leq C \int_{Q} k(x) v_{x}^{2} d x d a d t \\
& \leq C \int_{Q} k(x) e^{2 s \varphi} v_{x}^{2} d x d a d t+C s^{2} \int_{Q} \Theta^{2} e^{2 s \varphi} \frac{x^{2}}{k} v^{2} d x d a d t .
\end{aligned}
$$

If $M_{1} \geq 1$, using the Young's inequality to the function $v:=e^{s \varphi} v$, we have

$$
\begin{aligned}
\int_{Q}|\mu|^{2}|v|^{2} e^{2 s \varphi} d x d a d t & \leq\|\mu\|_{\infty}^{2} \int_{Q} v^{2} d x d a d t \\
& \leq C \int_{Q}\left(\frac{k^{1 / 3}}{x^{2 / 3}} v^{2}\right)^{3 / 4}\left(\frac{x^{2}}{k} v^{2}\right)^{1 / 4} d x d a d t \\
& \leq C \int_{Q} \frac{k^{1 / 3}}{x^{2 / 3}} v^{2} d x d a d t+C \int_{Q} \frac{x^{2}}{k} v^{2} d x d a d t .
\end{aligned}
$$

Now, consider the function $y(x)=\left(k(x) x^{4}\right)^{1 / 3}$. Clearly, $y(x)=k(x)\left(\frac{x^{2}}{k(x)}\right)^{2 / 3} \leq C k(x)$ and $\frac{k^{1 / 3}}{x^{2 / 3}}=\frac{y(x)}{x^{2}}$. Moreover, using Hypothesis 3.1, one has that the function $\frac{y(x)}{x^{q}}=\left(\frac{k(x)}{x^{\theta}}\right)^{\frac{1}{3}}$, where $q:=\frac{4+\vartheta}{3} \in(1,2)$, is nondecreasing near 0. The Hardy-Poincaré inequality (see [2, Proposition 2.1.]) implies

$$
\begin{aligned}
\int_{Q} \frac{k^{1 / 3}}{x^{2 / 3}} v^{2} d x d a d t & =\int_{Q} \frac{y}{x^{2}} v^{2} d x d a d t \leq C \int_{Q} y\left(v_{x}\right)^{2} d x d a d t \leq C \int_{Q} k(x) v_{x}^{2} d x d a d t \\
& \leq C \int_{Q} k(x) e^{2 s \varphi} v_{x}^{2} d x d a d t+C s^{2} \int_{Q} \Theta^{2} e^{2 s \varphi} \frac{x^{2}}{k} v^{2} d x d a d t .
\end{aligned}
$$

In any case, by (3.14), (3.15) and (3.16),

$$
\int_{Q}|\mu|^{2}|v|^{2} e^{2 s \varphi} d x d a d t \leq C \int_{Q} k(x) e^{2 s \varphi} v_{x}^{2} d x d a d t+C s^{2} \int_{Q} \Theta^{2} e^{2 s \varphi} \frac{x^{2}}{k} v^{2} d x d a d t .
$$

Using this last inequality in (3.13), it follows

$$
\begin{aligned}
\int_{Q}|\bar{f}|^{2} e^{2 s \varphi} d x d a d t & \leq 2 \int_{Q}|f|^{2} e^{2 s \varphi} d x d a d t+C \int_{Q} k(x) e^{2 s \varphi} v_{x}^{2} d x d a d t \\
& +C s^{2} \int_{Q} \Theta^{2} e^{2 s \varphi} \frac{x^{2}}{k} v^{2} d x d a d t \\
& \leq C \int_{Q}|f|^{2} e^{2 s \varphi} d x d a d t+C \int_{Q} \Theta k(x) e^{2 s \varphi} v_{x}^{2} d x d a d t \\
& +C s^{2} \int_{Q} \Theta^{3} e^{2 s \varphi} \frac{x^{2}}{k} v^{2} d x d a d t .
\end{aligned}
$$

Substituting in (3.12), one can conclude

$$
\begin{aligned}
& \int_{Q}\left(s \Theta k v_{x}^{2}+s^{3} \Theta^{3} \frac{x^{2}}{k} v^{2}\right) e^{2 s \varphi} d x d a d t \leq C\left(\int_{Q}|f|^{2} \frac{e^{2 s \varphi}}{k} d x d a d t\right. \\
& \left.+s \int_{0}^{T} \int_{0}^{A} \Theta(t, a)\left[k v_{x}^{2} e^{2 s \varphi}\right](t, a, 1) d a d t\right),
\end{aligned}
$$


for all $s$ large enough.

\subsubsection{Carleman inequalities when the degeneracy is at 1 .}

In this subsection we will consider the case when $k(1)=0$. Again $\mu$ satisfies (2.1) and on $k$ we make the following assumption:

Hypothesis 3.2. The function $k \in C^{0}[0,1] \cap C^{1}[0,1)$ is such that $k(1)=0, k>0$ on $[0,1)$ and there exists $M_{2} \in(0,2)$ such that $(x-1) k^{\prime}(x) \leq M_{2} k(x)$ for all $x \in[0,1]$. Moreover, if $M_{2} \leq 1$ one has to require that there exists $\theta \in\left(0, M_{2}\right]$, such that the function $x \mapsto \frac{k(x)}{|1-x|^{\theta}}$ is nonincreasing near 1 .

For Hypothesis 3.2 we can make the same considerations made for Hypothesis 3.1.

As in the previous subsection, let us introduce the weight function

$$
\bar{\varphi}(t, a, x):=\Theta(t, a)\left(\bar{p}(x)-2\|\bar{p}\|_{L^{\infty}(0,1)}\right),
$$

where $\Theta$ is as in (4.7) and $\bar{p}(x):=\int_{0}^{x} \frac{y-1}{k(y)} d y$. As before, $\bar{\varphi}(t, a, x)<0$ for all $(t, x) \in Q$ and $\bar{\varphi}(t, a, x) \rightarrow$ $-\infty$ as $t \rightarrow 0^{+}, T^{-}$or $a \rightarrow 0^{+}$. The following estimate holds:

Theorem 3.2. Assume that Hypothesis 3.2 is satisfied. Then, there exist two strictly positive constants $C$ and $s_{0}$ such that every solution $v$ of (3.1) in $v$ satisfies, for all $s \geq s_{0}$,

$$
\begin{aligned}
\int_{Q}\left(s k \Theta v_{x}^{2}+s^{3} \Theta^{3} \frac{(x-1)^{2}}{k} v^{2}\right) e^{2 s \bar{\varphi}} d x d a d t & \leq C \int_{Q} f^{2} e^{2 s \bar{\varphi}} d x d a d t \\
& +s C \int_{0}^{T} \int_{0}^{A} \Theta(t, a)\left[(1-x) v_{x}^{2} e^{2 s \bar{\varphi}}\right](t, a, 0) d a d t .
\end{aligned}
$$

The previous Carleman estimate holds for every function $v$ that satisfies $(3.1)$ in $(0, T) \times(0, A) \times(B, C)$ as long as $(0,1)$ is substituted by $(B, C)$ and $k$ satisfies Hypothesis 3.2 in $(B, C)$.

The proof of Theorem 3.2 is analogous to one of Theorem 3.1 so we omit it. However, we underline that in the proof of Theorem 3.1 we use [2, Lemma 3.1] which is proved only if $k$ degenerates at 0 ; actually we observe that the proof of [2, Lemma 3.1] does not depend on the degeneracy point; hence, it holds also if $k(1)=0$. Instead, Lemma 3.3, if $k(1)=0$, becomes

$$
-\int_{Q_{T, A}}\left[s \Theta(x-1) k w_{\chi}^{2}\right]_{0}^{1} d a d t=-\int_{Q_{T, A}}\left[s \Theta k w_{x}^{2}\right](t, a, 0) d a d t
$$

thus, Proposition 3.1 can be rewritten in the following way

$$
\begin{aligned}
& s C \int_{Q} \Theta k w_{x}^{2} d x d a d t+s^{3} C \int_{Q} \Theta^{3} \frac{(x-1)^{2}}{k} w^{2} d x d a d t \\
& \leq C\left(\int_{Q} f^{2} e^{2 s \bar{\varphi}} d x d a d t+\int_{Q_{T, A}}\left[s \Theta k w_{x}^{2}\right](t, a, 0) d a d t\right) .
\end{aligned}
$$

If $\mu \not \equiv 0$, we can proceed as in the proof of Theorem 3.1. However, while in that case we use the Hardy-Poincaré inequality proved in [2, Proposition 2.1] which holds only if $k(0)=0$, in this case we have to use the following inequality whose proof we postpone to the Appendix. 
Proposition 3.2 (Hardy-Poincaré inequalities). Assume that $k:[0,1] \longrightarrow \mathbb{R}_{+}$is in $\mathrm{C}([0,1]), k(1)=0, k>0$ on $[0,1)$

Case (i):

Hypothesis (HP1): Assume that $k$ is such that there exists $\theta \in(0,1)$ such that the function

$$
x \longrightarrow \frac{k(x)}{(1-x)^{\theta}} \text { is nondecreasing in neighbourhood of } x=1 \text {. }
$$

Then, there is a constant $C>0$ such that for any function $w$, locally absolutely continuous on [0, 1), continuous at 1 and satisfying

$$
w(1)=0, \text { and } \int_{0}^{1} k(x)\left|w^{\prime}(x)\right|^{2} d x<+\infty,
$$

the following inequality holds

$$
\int_{0}^{1} \frac{k(x)}{(1-x)^{2}} w^{2}(x) d x \leq C \int_{0}^{1} k(x)\left|w^{\prime}(x)\right|^{2} d x .
$$

If hypothesis (HP1) is replaced by

Hypothesis (HP1)': Assume that $k$ is such that there exists $\theta \in(0,1)$ such that the function

$$
x \longrightarrow \frac{k(x)}{(1-x)^{\theta}} \text { is nondecreasing in }[0,1),
$$

Then, for any function $w$, locally absolutely continuous on $[0,1)$, continuous at 1 and satisfying

$$
w(1)=0 \text {, and } \int_{0}^{1} k(x)\left|w^{\prime}(x)\right|^{2} d x<+\infty,
$$

the inequality (3.19) holds with the explicit constant $C=\frac{4}{(1-\theta)^{2}}$.

Case (ii):

Hypothesis (HP2): Assume that $k$ is such that there exists $\theta \in(1,2)$ such that the function

$$
x \longrightarrow \frac{k(x)}{(1-x)^{\theta}} \text { is nonincreasing in a neighbourhood of } x=1,
$$

Then, there is a constant $C>0$ such that for any function $w$, locally absolutely continuous on $[0,1)$ satisfying

$$
w(0)=0, \text { and } \int_{0}^{1} k(x)\left|w^{\prime}(x)\right|^{2} d x<+\infty,
$$

the inequality (3.19) holds.

If hypothesis (HP2) is replaced by

Hypothesis (HP2)': Assume that $k$ is such that there exists $\theta \in(1,2)$ such that the function

$$
x \rightarrow \frac{k(x)}{(1-x)^{\theta}} \text { is nonincreasing in }[0,1) .
$$

Then, for any function $w$, locally absolutely continuous on $[0,1)$ satisfying

$$
w(0)=0, \text { and } \int_{0}^{1} k(x)\left|w^{\prime}(x)\right|^{2} d x<+\infty,
$$

the inequality (3.19) holds with the explicit constant $C=\frac{4}{(1-\theta)^{2}}$. 


\section{Observability and controllability}

In this section we will prove, as a consequence of the Carleman estimates established in Section 3, observability inequalities for the associated adjoint problem of (1.1). From now on, we assume that the control set $\omega$ is such that

$$
\omega=(\alpha, \rho) \subset \subset(0,1) .
$$

Moreover, on $k$ and $\beta$ we assume the following assumptions:

Hypothesis 4.1. The function $k \in C^{0}[0,1] \cap C^{1}(0,1)$ is such that $k(0)=0=k(1), k>0$ on $(0,1)$ and there exist $M_{1}, M_{2} \in(0,2)$ such that $x k^{\prime}(x) \leq M_{1} k(x)$ and $(x-1) k^{\prime}(x) \leq M_{2} k(x)$ for all $x \in[0,1]$. Moreover, one has to require that:

1. if $M_{1} \geq 1$, there exists $\theta \in\left(0, M_{1}\right]$, such that the function $x \mapsto \frac{k(x)}{x^{\theta}}$ is nondecreasing near 0 ;

2. if $M_{2} \leq 1$, there exists $y \in\left(0, M_{2}\right]$, such that the function $x \mapsto \frac{k(x)}{|1-x|^{y}}$ is nonincreasing near 1 .

Hypothesis 4.2. Assume $T<A$ and suppose that there exists $\bar{a} \leq T$ such that

$$
\beta(a, x)=0 \text { for all }(a, x) \in[0, \bar{a}] \times[0,1] .
$$

Observe that Hypothesis 4.2 is the biological meaningful one. Indeed, $\bar{a}$ is the minimal age in which the female of the population become fertile, thus it is natural that before $\bar{a}$ there are no newborns. For other comments on Hypothesis 4.2 we refer to [13].

Under the previous hypotheses, the following observability inequality holds:

Proposition 4.1. Suppose that Hypotheses 3.1 or 3.2 or 4.1 and 4.2 hold. Then, for every $\delta \in(T, A)$, there exists a strictly positive constant $C=C(\delta)$ such that every solution $v \in U$ of

$$
\begin{cases}\frac{\partial v}{\partial t}+\frac{\partial v}{\partial a}+\left(k(x) v_{x}\right)_{x}-\mu(t, a, x) v+\beta(a, x) v(t, 0, x)=0, & (t, x, a) \in Q, \\ v(t, a, 0)=v(t, a, 1)=0, & (t, a) \in Q_{T, A}, \\ v(T, a, x)=v_{T}(a, x) \in L^{2}\left(Q_{A, 1}\right), & (a, x) \in Q_{A, 1} \\ v(t, A, x)=0, & (t, x) \in Q_{T, 1},\end{cases}
$$

satisfies

$$
\int_{0}^{A} \int_{0}^{1} v^{2}(T-\bar{a}, a, x) d x d a \leq C\left(\int_{0}^{\delta} \int_{0}^{1} v_{T}^{2}(a, x) d x d a+\int_{0}^{T} \int_{0}^{A} \int_{\omega} v^{2} d x d a d t\right) .
$$

Here $v_{T}(a, x)$ is such that $v_{T}(A, x)=0$ in $(0,1)$.

Remark 1. 1. If $T=\bar{a}$, the observability inequality given in the previous proposition is the corresponding of [1, Proposition 3.1], where the authors proved it under different assumptions and with $T \geq A$.

2. Moreover, as in [13], observe that in (4.4) the presence of the integral $\int_{0}^{\delta} \int_{0}^{1} v_{T}^{2}(a, x) d x d a$ is related to the presence of the term $\beta(a, x) v(t, 0, x)$ in the equation of (4.3). In fact, estimating such a term using the method of characteristic lines, we obtain the previous integral. Obviously, if $v_{T}(a, x)=0$ a.e. in $(0, \delta) \times$ $(0,1)$, we obtain the classical observability inequality.

Before proving Proposition 4.1 we will give some results that will be very helpful. As a first step we introduce the following class of functions

$$
\mathcal{W}:=\left\{v \text { solution of (4.3) } \mid v_{T} \in D\left(\mathcal{A}^{2}\right)\right\},
$$


where $D\left(\mathcal{A}^{2}\right)=\{u \in D(\mathcal{A}) \mid \mathcal{A} u \in D(\mathcal{A})\}$ is densely defined in $D(\mathcal{A})$ (see, for example, [6, Lemma 7.2]) and hence in $L^{2}\left(Q_{A, 1}\right)$. Obviously,

$$
\mathcal{W}=C^{1}([0, T] ; D(\mathcal{A})) \subset \mathcal{V}:=L^{2}\left(Q_{T, A} ; H_{k}^{2}(0,1)\right) \cap H^{1}\left(0, T ; H^{1}\left(0, A ; H_{k}^{1}(0,1)\right)\right) \subset \mathcal{U} .
$$

Proposition 4.2 (Caccioppoli's inequality). Let $\omega^{\prime}$ and $\omega$ two open subintervals of $(0,1)$ such that $\omega^{\prime} \subset \subset$ $\omega \subset \subset(0,1)$. Let $\psi(t, a, x):=\Theta(t, a) \Psi(x)$, where $\Theta$ is defined in (4.7) and $\Psi \in C^{1}(0,1)$ is a strictly negative function. Then, there exist two strictly positive constants $C$ and $s_{0}$ such that, for all $s \geq s_{0}$,

$$
\int_{0}^{T} \int_{0}^{A} \int_{\omega^{\prime}} v_{x}^{2} e^{2 s \psi} d x d a d t \leq C\left(\int_{0}^{T} \int_{0}^{A} \int_{\omega} v^{2} d x d a d t+\int_{Q} f^{2} e^{2 s \psi} d x d a d t\right),
$$

for every solution $v$ of (3.1).

The proof of the previous proposition is similar to the one given in [13], but we repeat it in the Appendix for the reader's convenience.

Moreover, the following non degenerate inequality proved in [13] is crucial:

Theorem 4.1. [see [13, Theorem 3.2]] Let $z \in Z$ be the solution of (3.1), where $f \in L^{2}(Q), k \in C^{1}([0,1])$ is a strictly positive function and

$$
Z:=L^{2}\left(Q_{T, A} ; H^{2}(0,1) \cap H_{0}^{1}(0,1)\right) \cap H^{1}\left(0, T ; H^{1}\left(0, A ; H_{0}^{1}(0,1)\right)\right) .
$$

Then, there exist two strictly positive constants $C$ and $s_{0}$, such that, for any $s \geq s_{0}, z$ satisfies the estimate

$$
\int_{Q}\left(s^{3} \phi^{3} z^{2}+s \phi z_{x}^{2}\right) e^{2 s \Phi} d x d a d t \leq C\left(\int_{Q} f^{2} e^{2 s \Phi} d x d a d t-s \kappa \int_{0}^{T} \int_{0}^{A}\left[k e^{2 s \Phi} \phi\left(z_{x}\right)^{2}\right]_{x=0}^{x=1} d a d t\right),
$$

where the functions $\phi$ and $\Phi$ are defined as follows

$$
\begin{gathered}
\phi(t, a, x)=\Theta(t, a) e^{\kappa \sigma(x)}, \quad \Theta(t, a)=\frac{1}{t^{4}(T-t)^{4} a^{4}}, \\
\Phi(a, t, x)=\Theta(t, a) \Psi(x), \quad \Psi(x)=e^{\kappa \sigma(x)}-e^{2 \kappa\|\sigma\|_{\infty}}, \\
(t, a, x) \in Q, \kappa>0 \text { and } \sigma(x):=\mathfrak{d} \int_{x}^{1} \frac{1}{k(t)} d t, \text { where } \mathfrak{d}=\left\|k^{\prime}\right\|_{L^{\infty}(0,1)} .
\end{gathered}
$$

Remark 2. The previous Theorem still holds under the weaker assumption $k \in W^{1, \infty}(0,1)$ without any additional assumption.

On the other hand, if we require $k \in W^{1,1}(0,1)$ then we have to add the following hypothesis: there exist two functions $\mathfrak{g} \in L^{1}(0,1), \mathfrak{h} \in W^{1, \infty}(0,1)$ and two strictly positive constants $\mathfrak{g}_{0}, \mathfrak{h}_{0}$ such that $\mathfrak{g}(x) \geq \mathfrak{g}_{0}$ and

$$
-\frac{k^{\prime}(x)}{2 \sqrt{k(x)}}\left(\int_{x}^{1} \mathfrak{g}(t) d t+\mathfrak{h}_{0}\right)+\sqrt{k(x)} \mathfrak{g}(x)=\mathfrak{h}(x) \quad \text { for a.e. } x \in[0,1] .
$$

In this case, i.e. if $k \in W^{1,1}(0,1)$, the function $\Psi$ in (4.7) becomes

$$
\Psi(x):=-r\left[\int_{0}^{x} \frac{1}{\sqrt{k(t)}} \int_{t}^{1} \mathfrak{g}(s) d s d t+\int_{0}^{x} \frac{\mathfrak{h}_{0}}{\sqrt{k(t)}} d t\right]-\mathfrak{c},
$$

where $r$ and $\mathfrak{c}$ are suitable strictly positive functions. For other comments on Theorem 4.1 we refer to [13].

With the aid of Theorems 3.1, 3.2, 4.1 and Proposition 4.2, we can now show $\omega$-local Carleman estimates for (3.1). 
Theorem 4.2. Assume Hypothesis 3.1. Then, there exist two strictly positive constants $C$ and $s_{0}$ such that every solution $v$ of (3.1) in $v$ satisfies, for all $s \geq s_{0}$,

$$
\int_{Q}\left(s \Theta k v_{X}^{2}+s^{3} \Theta^{3} \frac{x^{2}}{k} v^{2}\right) e^{2 s \varphi} d x d a d t \leq C\left(\int_{Q} f^{2} d x d a d t+\int_{0}^{T} \int_{0}^{A} \int_{\omega} v^{2} d x d a d t\right) .
$$

Proof. Let us consider a smooth function $\xi:[0,1] \rightarrow \mathbb{R}$ such that

$$
\begin{cases}0 \leq \xi(x) \leq 1, & \text { for all } x \in[0,1] \\ \xi(x)=1, & x \in[0,(2 \alpha+\rho) / 3] \\ \xi(x)=0, & x \in[(\alpha+2 \rho) / 3,1]\end{cases}
$$

We define $w(t, a, x):=\xi(x) v(t, a, x)$ where $v \in \mathcal{V}$ satisfies (3.1). Then $w$ satisfies

$$
\begin{cases}w_{t}+w_{a}+\left(k w_{x}\right)_{x}-\mu w=\xi f+\left(k \xi_{x} v\right)_{x}+\xi_{x} k v_{x}=: h, & (t, a, x) \in Q, \\ w(t, a, 0)=w(t, a, 1)=0, & (t, a) \in Q_{T, A} .\end{cases}
$$

Thus, applying Theorem 3.1 and Proposition 4.2,

$$
\begin{aligned}
& \int_{0}^{T} \int_{0}^{A} \int_{0}^{\frac{2 \alpha+\rho}{3}}\left(s \Theta k v_{x}^{2}+s^{3} \Theta^{3} \frac{x^{2}}{k} v^{2}\right) e^{2 s \varphi} d x d a d t \\
& =\int_{0}^{T} \int_{0}^{A} \int_{0}^{\frac{2 \alpha+\rho}{3}}\left(s \Theta k w_{x}^{2}+s^{3} \Theta^{3} \frac{x^{2}}{k} w^{2}\right) e^{2 s \varphi} d x d a d t \\
& \leq \int_{Q}\left(s \Theta k w_{x}^{2}+s^{3} \Theta^{3} \frac{x^{2}}{k} w^{2}\right) e^{2 s \varphi} d x d a d t \leq C \int_{Q} h^{2} e^{2 s \varphi} d x d a d t \\
& \leq C\left(\int_{Q} f^{2} e^{2 s \varphi} d x d a d t+\int_{0}^{T} \int_{0}^{A} \int_{\omega^{\prime}} v^{2} d x d a d t+\int_{0}^{T} \int_{0}^{A} \int_{\omega^{\prime}} v_{x}^{2} e^{2 s \varphi} d x d a d t\right) \\
& \leq C\left(\int_{Q}^{T} f^{2} e^{2 s \varphi} d x d a d t+\int_{0}^{A} \int_{0} \int_{\omega} v^{2} d x d a d t\right),
\end{aligned}
$$

where $\omega^{\prime}:=\left(\frac{2 \alpha+\rho}{3}, \frac{\alpha+2 \rho}{3}\right)$.

Now, consider $z=\eta v$, where $\eta=1-\xi$ and take $\bar{\alpha} \in(0, \alpha)$. Then $z$ satisfies

$$
\begin{cases}z_{t}+z_{a}+\left(k z_{x}\right)_{x}-\mu z=\eta f+\left(k \eta_{x} v\right)_{x}+\eta_{x} k v_{x}=: h, & (t, a, x) \in Q_{T, A} \times(\bar{\alpha}, 1)=: \bar{Q}, \\ z(t, a, \bar{\alpha})=z(t, a, 1)=0, & (t, a) \in Q_{T, A} .\end{cases}
$$

Clearly the equation satisfied by $z$ is not degenerate, thus applying Theorem 4.1 and Proposition 4.2, one has

$$
\begin{aligned}
& \int_{\bar{Q}}\left(s^{3} \phi^{3} z^{2}+s \phi z_{\chi}^{2}\right) e^{2 s \Phi} d x d a d t \leq C \int_{\bar{Q}} h^{2} e^{2 s \Phi} d x d a d t \\
& \leq C\left(\int_{\bar{Q}} f^{2} e^{2 s \Phi} d x d a d t+\int_{0}^{T} \int_{0}^{A} \int_{\omega^{\prime}}\left(v^{2}+v_{x}^{2}\right) e^{2 s \Phi} d x d a d t\right) \\
& \leq C\left(\int_{Q} f^{2} e^{2 s \Phi} d x d a d t+\int_{0}^{T} \int_{0}^{A} \int_{\omega} v^{2} d x d a d t\right) .
\end{aligned}
$$


Hence

$$
\begin{aligned}
& \int_{0}^{T} \int_{0}^{T} \int_{\frac{\alpha+2 \rho}{3}}^{1}\left(s^{3} \phi^{3} v^{2}+s \phi v_{x}^{2}\right) e^{2 s \Phi} d x d a d t=\int_{0}^{T} \int_{0}^{A} \int_{\frac{\alpha+2 \rho}{3}}^{1}\left(s^{3} \phi^{3} z^{2}+s \phi z_{x}^{2}\right) e^{2 s \Phi} d x d a d t \\
& \leq C\left(\int_{Q} f^{2} e^{2 s \Phi} d x d a d t+\int_{0}^{T} \int_{0}^{A} \int_{\omega} v^{2} d x d a d t\right),
\end{aligned}
$$

for a strictly positive constant $C$. Proceeding, for example, as in [16] one can prove the existence of $\varsigma>0$, such that, for all $(t, a, x) \in[0, T] \times[0, A] \times[\bar{\alpha}, 1]$, we have

$$
e^{2 s \varphi} \leq \varsigma e^{2 s \Phi}, \frac{x^{2}}{k(x)} e^{2 s \varphi} \leq \varsigma e^{2 s \Phi} .
$$

Thus, for a strictly positive constant $C$,

$$
\begin{aligned}
& \int_{0}^{T} \int_{0}^{A} \int_{\frac{\alpha+2 \rho}{3}}^{1}\left(s \Theta k v_{x}^{2}+s^{3} \Theta^{3} \frac{x^{2}}{k} v^{2}\right) e^{2 s \varphi} d x d a d t \\
& \leq C\left(\int_{0}^{T} \int_{0}^{A} \int_{\frac{\alpha+2 \rho}{3}}^{1}\left(s^{3} \phi^{3} v^{2}+s \phi v_{x}^{2}\right) e^{2 s \Phi} d x d a d t\right) \\
& \leq C\left(\int_{Q} f^{2} e^{2 s \Phi} d x d a d t+\int_{0}^{T} \int_{0}^{A} \int_{\omega} v^{2} d x d a d t\right) .
\end{aligned}
$$

Now, consider $\tilde{\alpha} \in(\alpha,(2 \alpha+\rho) / 3), \tilde{\rho} \in((\alpha+2 \rho) / 3, \rho)$ and a smooth function $\tau:[0,1] \rightarrow \mathbb{R}$ such that

$$
\begin{cases}0 \leq \tau(x) \leq 1, & \text { for all } x \in[0,1], \\ \tau(x)=1, & x \in[(2 \alpha+\rho) / 3,(\alpha+2 \rho) / 3], \\ \tau(x)=0, & x \in[0, \tilde{\alpha}] \cup[\tilde{\rho}, 1],\end{cases}
$$

and define $\zeta(t, a, x):=\tau(x) v(t, a, x)$. Clearly, $\zeta$ satisfies (4.10) with $h:=\tau f+\left(k \tau_{x} v\right)_{x}+\tau_{x} k v_{x}$. Observe that in this case $\tau_{x}, \tau_{x x} \not \equiv 0$ in $\bar{\omega}:=\left(\tilde{\alpha}, \frac{2 \alpha+\rho}{3}\right) \cup\left(\frac{\alpha+2 \rho}{3}, \tilde{\rho}\right)$. As before, by Theorem 4.1, Proposition 4.2 and (4.11), we have

$$
\begin{aligned}
& \int_{0}^{T} \int_{0}^{T} \int_{\frac{2 \alpha+\rho}{3}}^{\frac{\alpha+2 \rho}{3}}\left(s \Theta k v_{x}^{2}+s^{3} \Theta^{3} \frac{x^{2}}{k} v^{2}\right) e^{2 s \varphi} d x d a d t \\
& \leq C\left(\int_{0}^{T} \int_{0}^{A} \int_{\frac{2 \alpha+\rho}{3}}^{\frac{\alpha+2 \rho}{3}}\left(s^{3} \phi^{3} v^{2}+s \phi v_{x}^{2}\right) e^{2 s \Phi} d x d a d t\right) \\
& =C\left(\int_{0}^{T} \int_{0}^{\left.\frac{A}{3} \int_{\frac{2 \alpha+\rho}{3}}^{\frac{\alpha+2 \rho}{3}}\left(s^{3} \phi^{3} \zeta^{2}+s \phi \zeta_{x}^{2}\right) e^{2 s \Phi} d x d a d t\right)}\right. \\
& \leq C\left(\int_{Q} f^{2} e^{2 s \Phi} d x d a d t+\int_{0}^{T} \int_{0}^{A} \int_{\omega} v^{2} d x d a d t\right) .
\end{aligned}
$$

Adding (4.9), (4.12) and (4.13), the thesis follows.

Proceeding as before one can prove 
Theorem 4.3. Assume Hypothesis 3.2. Then, there exist two strictly positive constants $C$ and $s_{0}$ such that every solution $v$ of (3.1) in $v$ satisfies, for all $s \geq s_{0}$,

$$
\int_{Q}\left(s \Theta k v_{x}^{2}+s^{3} \Theta^{3} \frac{(1-x)^{2}}{k} v^{2}\right) e^{2 s \varphi} d x d a d t \leq C\left(\int_{Q} f^{2} d x d a d t+\int_{0}^{T} \int_{0}^{A} \int_{\omega}^{A} v^{2} d x d a d t\right) .
$$

Remark 3. Observe that the results of Theorems 4.2 and 4.3 still hold true if we substitute the domain $(0, T) \times$ $(0, A)$ with a general domain $\left(T_{1}, T_{2}\right) \times(y, A)$, provided that $\mu$ and $\beta$ satisfy the required assumptions. In this case, in place of the function $\Theta$ defined in (4.7), we have to consider the weight function

$$
\tilde{\Theta}(t, a):=\frac{1}{\left(t-T_{1}\right)^{4}\left(T_{2}-t\right)^{4}(a-y)^{4}} .
$$

Using the previous local Carleman estimates one can prove the next observability inequalities.

Theorem 4.4. Assume Hypotheses 3.1 or 3.2 and 4.2 with $T>\bar{a}$. Then, for every $\delta \in(0, A)$, there exists $a$ strictly positive constant $C=C(\delta)$ such that every solution $v$ of (4.3) in $v$ satisfies

$$
\begin{aligned}
\int_{0}^{A} \int_{0}^{1} v^{2}(T-\bar{a}, a, x) d x d a & \leq C \int_{0}^{T} \int_{0}^{\delta} \int_{0}^{1} v^{2}(t, a, x) d x d a d t \\
& +C\left(\int_{0}^{\bar{a}} \int_{0}^{1} v_{T}^{2}(a, x) d x d a+\int_{0}^{T} \int_{0}^{A} \int_{\omega} v^{2} d x d a d t\right) .
\end{aligned}
$$

Moreover, if $v_{T}(a, x)=0$ for all $(a, x) \in(0, \bar{a}) \times(0,1)$, one has

$$
\int_{0}^{A} \int_{0}^{1} v^{2}(T-\bar{a}, a, x) d x d a \leq C\left(\int_{0}^{T} \int_{0}^{\delta} \int_{0}^{1} v^{2}(t, a, x) d x d a d t+\int_{0}^{T} \int_{0}^{A} \int_{\omega} v^{2} d x d a d t\right) .
$$

Proof. As in [13] and using the method of characteristic lines, one can prove the following implicit formula for $v$ solution of (4.3):

$$
S(T-t) v_{T}(T+a-t, \cdot)
$$

if $t \geq \tilde{T}+a$ and

$$
v(t, a, \cdot)= \begin{cases}S(T-t) v_{T}(T+a-t, \cdot)+\int_{a}^{T+a-t} S(s-a) \beta(s, \cdot) v(s+t-a, 0, \cdot) d s, & \Gamma=\bar{a} \\ \int_{a}^{A} S(s-a) \beta(s, \cdot) v(s+t-a, 0, \cdot) d s, & \Gamma=\Gamma_{A, T},\end{cases}
$$

otherwise. Here $(S(t))_{t \geq 0}$ is the semigroup generated by the operator $\mathcal{A}_{0}-\mu I d$ for all $u \in D\left(\mathcal{A}_{0}\right)$ (Id is the identity operator), $\Gamma_{A, T}:=A-a+t-\tilde{T}$ and

$$
\Gamma:=\min \left\{\bar{a}, \Gamma_{A, T}\right\} .
$$

In particular, it results

$$
v(t, 0, \cdot):=S(T-t) v_{T}(T-t, \cdot)
$$

if $t \geq T-\bar{a}$.

Proceeding as in [13, Theorem 4.4], with suitable changes, one has that there exists a positive constant $C$ such that:

$$
\int_{Q_{A, 1}} v^{2}(\tilde{T}, a, x) d x d a \leq C \int_{T-\frac{\bar{a}}{2}}^{T-\frac{\bar{a}}{4}} \int_{Q_{A, 1}} v^{2}(t, a, x) d x d a d t .
$$

However, here we make all the calculations in order to make precise some steps in [13, Theorem 4.4], where there is a misprint. Indeed in (4.19) the integration is made on $\left[T-\frac{\bar{a}}{2}, T-\frac{\bar{a}}{4}\right]$ in place of $\left[\frac{T}{4}, \frac{3 T}{4}\right]$ as in [13]. 
The integration on $\left[\frac{T}{4}, \frac{3 T}{4}\right]$ is correct if $T=\bar{a}$ as in the next Corollary or in [13, Corollary 4.1]. Indeed, define, for $\varsigma>0$, the function $w=e^{\varsigma t} v$, where $v$ solves (4.3). Then $w$ satisfies

$$
\begin{cases}\frac{\partial w}{\partial t}+\frac{\partial w}{\partial a}+\left(k(x) w_{x}\right)_{x}-(\mu(t, a, x)+\varsigma) w=-\beta(a, x) w(t, 0, x), & (t, x, a) \in \tilde{Q}, \\ w(t, a, 0)=w(t, a, 1)=0, & (t, a) \in \tilde{Q}_{T, A}, \\ w(T, a, x)=e^{\varsigma T} v_{T}(a, x), & (a, x) \in Q_{A, 1}, \\ w(t, A, x)=0, & (t, x) \in \tilde{Q}_{T, 1},\end{cases}
$$

where $\tilde{Q}:=(\tilde{T}, T) \times Q_{A, 1}, \tilde{Q}_{T, A}:=(\tilde{T}, T) \times(0, A)$ and $\tilde{Q}_{T, 1}:=(\tilde{T}, T) \times(0,1)$. Multiplying the equation of (4.20) by $-w$ and integrating by parts on $Q_{t}:=(\tilde{T}, t) \times(0, A) \times(0,1)$, it results

$$
\begin{aligned}
& -\frac{1}{2} \int_{Q_{A, 1}} w^{2}(t, a, x) d x d a+\frac{e^{\varsigma \tilde{T}}}{2} \int_{Q_{A, 1}} v^{2}(\tilde{T}, a, x) d x d a+\frac{1}{2} \int_{\tilde{T}}^{t} \int_{0}^{1} w^{2}(\tau, 0, x) d x d \tau \\
& +\varsigma \int_{Q_{t}} w^{2}(\tau, a, x) d x d a d \tau \leq \int_{Q_{t}} \beta w(\tau, 0, x) w d x d a d \tau \\
& \leq\|\beta\|_{L^{\infty}(Q)} \frac{1}{\epsilon} \int_{Q_{t}} w^{2} d x d a d \tau+\epsilon A\|\beta\|_{L^{\infty}(Q)} \int_{\tilde{T}}^{t} \int_{0}^{1} w^{2}(\tau, 0, x) d x d \tau,
\end{aligned}
$$

for $\epsilon>0$. Choosing $\epsilon=\frac{1}{2\|\beta\|_{L^{\infty}(Q)} A}$ and $\varsigma=\frac{\|\beta\|_{L^{\infty}(Q)}}{\epsilon}$, we have

$$
\int_{Q_{A, 1}} v^{2}(\tilde{T}, a, x) d x d a \leq C \int_{Q_{A, 1}} w^{2}(t, a, x) d x d a \leq C \int_{Q_{A, 1}} v^{2}(t, a, x) d x d a .
$$

Then, integrating over $\left[T-\frac{\bar{a}}{2}, T-\frac{\bar{a}}{4}\right]$, we have (4.19).

Now, take $\delta \in(0, A)$. By (4.19), we have

$$
\int_{Q_{A, 1}} v^{2}(\tilde{T}, a, x) d x d a \leq C \int_{T-\frac{\bar{a}}{2}}^{T-\frac{\bar{a}}{4}}\left(\int_{0}^{\delta}+\int_{\delta}^{A}\right) \int_{0}^{1} v^{2}(t, a, x) d x d a d t .
$$

Consider the term $\int_{T-\frac{\bar{a}}{2}}^{T-\frac{\bar{a}}{4}} \int_{\delta}^{A} \int_{0}^{1} v^{2}(t, a, x) d x d a d t$. If Hypothesis 3.1 holds, proceeding as in [2, Lemma 3.2], one has

$$
\int_{0}^{1} v^{2} d x \leq C\left(\int_{0}^{1} k v_{x}^{2} d x+\int_{0}^{1} \frac{x^{2}}{k} v^{2} d x\right)
$$

for a strictly positive constant $C$. Hence,

$$
\begin{aligned}
\int_{T-\frac{\bar{\alpha}}{2}}^{T-\frac{\bar{\alpha}}{4}} \int_{\delta}^{A} \int_{0}^{1} v^{2}(t, a, x) d x d a d t & \leq C \int_{T-\frac{\bar{\alpha}}{2}}^{T-\frac{\bar{\alpha}}{4}} \int_{\delta}^{A} \int_{0}^{1} \tilde{\Theta} k v_{x}^{2} e^{2 s \tilde{\varphi}} d x d a d t \\
& +C \int_{T-\frac{\bar{\alpha}}{2}}^{T-\frac{\bar{\alpha}}{4}} \int_{\delta}^{A} \int_{0}^{1} \tilde{\Theta}^{3} \frac{x^{2}}{k} v^{2} e^{2 s \tilde{\varphi}} d x d a d t,
\end{aligned}
$$


where $\tilde{\Theta}$ is defined in (4.14) with $T_{1}:=T-\bar{a}, T_{2}:=T, y=0$ and $\tilde{\varphi}$ is the function associated to $\tilde{\Theta}$ according to (3.2). Analogously, if Hypothesis 3.2 holds, then we obtain

$$
\begin{aligned}
\int_{T-\frac{\bar{\alpha}}{2}}^{T-\frac{\bar{a}}{4}} \int_{\delta}^{A} \int_{0}^{1} v^{2}(t, a, x) d x d a d t & \leq C \int_{T-\frac{\bar{a}}{2}}^{T-\frac{\bar{a}}{4}} \int_{\delta}^{A} \int_{0}^{1} \tilde{\Theta} k v_{x}^{2} e^{2 s \tilde{\tilde{\varphi}}} d x d a d t \\
& +C \int_{T-\frac{\bar{\alpha}}{2}}^{T-\frac{\bar{\alpha}}{4}} \int_{\delta}^{A} \int_{0}^{1} \tilde{\Theta}^{3} \frac{(1-x)^{2}}{k} v^{2} e^{2 s \tilde{\bar{\varphi}}} d x d a d t
\end{aligned}
$$

where $\tilde{\tilde{\varphi}}$ is the function associated to $\tilde{\Theta}$ according to (3.18). Thus, by Theorem 4.2 or 4.3 applied to $\bar{Q}:=$ $(T-\bar{a}, T) \times(0, A) \times(0,1)$ and Remark 3 ,

$$
\int_{T-\frac{\bar{\alpha}}{2}}^{T-\frac{\bar{\alpha}}{4}} \int_{\delta}^{A} \int_{0}^{1} v^{2}(t, a, x) d x d a d t \leq C\left(\int_{\bar{Q}} f^{2} d x d a d t+\int_{0}^{T} \int_{0}^{A} \int_{\omega} v^{2} d x d a d t\right),
$$

where, in this case, $f(t, a, x):=-\beta(a, x) v(t, 0, x)$. Thus

$$
\int_{T-\frac{\bar{a}}{2}}^{T-\frac{\bar{a}}{4}} \int_{\delta}^{A} \int_{0}^{1} v^{2}(t, a, x) d x d a d t \leq C\|\beta\|_{L^{\infty}(Q)}^{2}\left(\int_{\bar{Q}} v^{2}(t, 0, x) d x d a d t+\int_{0}^{T} \int_{0}^{A} \int_{\omega}^{A} v^{2} d x d a d t\right),
$$

for a strictly positive constant $C$. By (4.18), (4.24) and proceeding as in [13], we have

$$
\int_{T-\frac{\bar{\alpha}}{2}}^{T-\frac{\bar{a}}{4}} \int_{\delta}^{A} \int_{0}^{1} v^{2}(t, a, x) d x d a d t \leq C\|\beta\|_{L^{\infty}(Q)}^{2}\left(\int_{Q_{\bar{a}, 1}} v_{T}^{2}(a, x) d x d a+\iint_{0}^{T} \int_{0}^{A} \int_{\omega}^{A} v^{2} d x d a d t\right),
$$

for a strictly positive constant $C$. By (4.22) and (4.25), it results

$$
\begin{aligned}
\int_{Q_{A, 1}} v^{2}(\tilde{T}, a, x) d x d a & \leq C \int_{0}^{T} \int_{0}^{\delta} \int_{0}^{1} v^{2}(t, a, x) d x d a d t \\
& +C\left(\int_{Q_{a, 1}} v_{T}^{2}(a, x) d x d a+\int_{0}^{T} \int_{0}^{A} \int_{\omega}^{A} v^{2} d x d a d t\right) .
\end{aligned}
$$

Corollary 4.1. Assume $\bar{a}=T$, Hypotheses 3.1 or 3.2 and 4.2. Then, for every $\delta \in(0, A)$, there exists a strictly positive constant $C=C(\delta)$ such that every solution $v$ of (4.3) in $\mathcal{v}$ satisfies

$$
\begin{aligned}
\int_{0}^{A} \int_{0}^{1} v^{2}(0, a, x) d x d a & \leq C \int_{0}^{T} \int_{0}^{\delta} \int_{0}^{1} v^{2}(t, a, x) d x d a d t \\
& +C\left(\int_{0}^{\bar{a}} \int_{0}^{1} v_{T}^{2}(a, x) d x d a+\int_{0}^{T} \int_{0}^{A} \int_{\omega} v^{2} d x d a d t\right) .
\end{aligned}
$$

Moreover, if $v_{T}(a, x)=0$ for all $(a, x) \in(0, \bar{a}) \times(0,1)$, one has

$$
\int_{0}^{A} \int_{0}^{1} v^{2}(0, a, x) d x d a \leq C\left(\int_{0}^{T} \int_{0}^{\delta} \int_{0}^{1} v^{2}(t, a, x) d x d a d t+\int_{0}^{T} \int_{0}^{A} \int_{\omega} v^{2} d x d a d t\right) .
$$


Actually, proceeding as in [13] with suitable changes, we can improve the previous results in the following way:

Theorem 4.5. Assume Hypotheses 3.1 or 3.2 and 4.2. Then, for every $\delta \in(T, A)$, there exists a strictly positive constant $C=C(\delta)$ such that every solution $v$ of (4.3) in $v$ satisfies

$$
\int_{0}^{A} \int_{0}^{1} v^{2}(T-\bar{a}, a, x) d x d a \leq C\left(\int_{0}^{\delta} \int_{0}^{1} v_{T}^{2}(a, x) d x d a+\int_{0}^{T} \int_{0}^{A} \int_{\omega} v^{2} d x d a d t\right) .
$$

By Theorem 4.5 and using a density argument, one can deduce Proposition 4.1. As a consequence one can prove, as in [13], the following null controllability results:

Theorem 4.6. Assume Hypotheses 3.1 or 3.2 or 4.1 and 4.2. Then, given $T>0$ and $y_{0} \in L^{2}\left(Q_{A, 1}\right)$, for every $\delta \in(T, A)$, there exists a control $f_{\delta} \in L^{2}(\tilde{Q})$ such that the solution $y_{\delta} \in \mathcal{U}$ of

$$
\begin{cases}\frac{\partial y}{\partial t}+\frac{\partial y}{\partial a}-\left(k(x) y_{x}\right)_{x}+\mu(t, a, x) y=f_{\delta}(t, x, a) \chi_{\omega} & \text { in } \tilde{Q}, \\ y(t, a, 1)=y(t, a, 0)=0 & \text { on } \tilde{Q}_{T, A}, \\ y(\tilde{T}, a, x)=y_{0}(a, x) & \text { in } Q_{A, 1}, \\ y(t, 0, x)=\int_{0}^{A} \beta(a, x) y(t, a, x) d a & \text { in } \tilde{Q}_{T, 1},\end{cases}
$$

satisfies

$$
y_{\delta}(T, a, x)=0 \quad \text { a.e. }(a, x) \in(\delta, A) \times(0,1) .
$$

Moreover, there exists $C=C(\delta)>0$ such that

$$
\left\|f_{\delta}\right\|_{L^{2}(\tilde{Q})} \leq C\left\|y_{0}\right\|_{L^{2}\left(Q_{A, 1}\right)} .
$$

Here, we recall, $\tilde{Q}=(\tilde{T}, T) \times(0, A) \times(0,1), \tilde{Q}_{T, A}=(\tilde{T}, T) \times(0, A)$ and $\tilde{Q}_{T, 1}=(\tilde{T}, T) \times(0,1)$.

Observe that if $T=\bar{a}$, Theorem 4.6 is exactly the null controllability result that we expect. Indeed, in this case (4.27) coincide with (1.1). On the other hand, if $T>\bar{a}$, the null controllability for (1.1) is given in the next theorem and it is based on the previous result:

Theorem 4.7. Assume Hypotheses 3.1 or 3.2 and 4.2. Then, given $T \in(0, A)$ and $y_{0} \in L^{2}\left(Q_{A, 1}\right)$, for every $\delta \in(T, A)$, there exists a control $f_{\delta} \in L^{2}(Q)$ such that the solution $y_{\delta}$ of (1.1) satisfies

$$
y_{\delta}(T, a, x)=0 \quad \text { a.e. }(a, x) \in(\delta, A) \times(0,1) .
$$

Moreover, there exists $C=C(\delta)>0$ such that

$$
\left\|f_{\delta}\right\|_{L^{2}(Q)} \leq C\left\|y_{0}\right\|_{L^{2}\left(Q_{A, 1}\right)} .
$$

Proof. The proof is similar to the one of [13, Theorem 4.8]. However, here we make all the calculations in order to make precise some steps in [13, Theorem 4.8], where there is a misprint and a term was missing.

As a first step, set $\tilde{T}:=T-\bar{a} \in(0, T)$. By Theorem 2.1, there exists a unique solution $u$ of

$$
\begin{cases}\frac{\partial u}{\partial t}+\frac{\partial u}{\partial a}-\left(k(x) u_{x}\right)_{x}+\mu(t, a, x) u=0 & \text { in }(0, \tilde{T}) \times(0, A) \times(0,1), \\ u(t, a, 1)=u(t, a, 0)=0 & \text { on }(0, \tilde{T}) \times(0, A), \\ u(0, a, x)=y_{0}(a, x) & \text { in }(0, A) \times(0,1), \\ u(t, 0, x)=\int_{0}^{A} \beta(a, x) u(t, a, x) d a & \text { in }(0, \tilde{T}) \times(0,1) .\end{cases}
$$


Set $\tilde{y}_{0}(a, x):=u(\tilde{T}, a, x)$; clearly $\tilde{y}_{0} \in L^{2}\left(Q_{A, 1}\right)$. Now, consider

$$
\begin{cases}\frac{\partial w}{\partial t}+\frac{\partial w}{\partial a}-\left(k(x) w_{x}\right)_{x}+\mu(t, a, x) w=h(t, x, a) \chi_{\omega} & \text { in } \tilde{Q}, \\ w(t, a, 1)=w(t, a, 0)=0 & \text { on } \tilde{Q}_{T, A}, \\ w(\tilde{T}, a, x)=\tilde{y}_{0}(a, x) & \text { in } Q_{A, 1}, \\ w(t, 0, x)=\int_{0}^{A} \beta(a, x) w(t, a, x) d a & \text { in } \tilde{Q}_{T, 1} .\end{cases}
$$

Again, by Theorem 2.1, there exists a unique solution $w_{\delta}$ of (4.31) and, by the previous Theorem, there exists a control $h_{\delta} \in L^{2}(\tilde{Q})$ such that

$$
w_{\delta}(T, a, x)=0 \text { a.e. }(a, x) \in(\delta, A) \times(0,1)
$$

and

$$
\left\|h_{\delta}\right\|_{L^{2}(\tilde{Q})} \leq C\left\|\tilde{y}_{0}\right\|_{L^{2}\left(Q_{A, 1}\right)}
$$

for a positive constant $C$.

Now, define $y_{\delta}$ and $f_{\delta}$ by

$$
y_{\delta}:=\left\{\begin{array}{ll}
u, & \text { in }[0, \tilde{T}], \\
w_{\delta}, & \text { in }[\tilde{T}, T]
\end{array} \text { and } f_{\delta}:= \begin{cases}0, & \text { in }[0, \tilde{T}], \\
h_{\delta}, & \text { in }[\tilde{T}, T] .\end{cases}\right.
$$

Then $y_{\delta}$ satisfies (1.1) and $f_{\delta} \in L^{2}(Q)$ is such that

$$
y_{\delta}(T, a, x)=0 \text { a.e. }(a, x) \in(\delta, A) \times(0,1) .
$$

Indeed $y_{\delta}(T, a, x)=w_{\delta}(T, a, x)=0$ a.e. $(a, x) \in(\delta, A) \times(0,1)$.

Now, we prove (4.29). As a first step, as in [13], we multiply the equation of (4.30) by $u$. Then, integrating over $Q_{A, 1}$, we obtain:

$$
\begin{aligned}
\frac{1}{2} \frac{d}{d t} \int_{0}^{A} \int_{0}^{1} u^{2} d x d a & +\frac{1}{2} \int_{0}^{1} u^{2}(t, A, x) d x+\int_{0}^{A} \int_{0}^{1} k u_{x}^{2} d x d a+\int_{0}^{A} \int_{0}^{1} \mu u^{2} d x d a \\
& =\frac{1}{2} \int_{0}^{1} u^{2}(t, 0, x) d x .
\end{aligned}
$$

Hence, using the fact that $u(t, 0, x)=\int_{0}^{A} \beta(a, x) u(t, a, x) d a$, we have

$$
\frac{1}{2} \frac{d}{d t} \int_{0}^{A} \int_{0}^{1} u^{2} d x d a \leq \frac{1}{2} \int_{0}^{1}\left(\int_{0}^{A} \beta(a, x) u(t, a, x) d a\right)^{2} d x \leq \frac{C}{2} \int_{0}^{A} \int_{0}^{1} u^{2} d x d a .
$$

Setting $F(t):=\|u(t)\|_{L^{2}\left(Q_{A, 1}\right)}^{2}$ and multiplying the previous inequality by $e^{-C t}$, it results

$$
\frac{d}{d t}\left(e^{-C t} F(t)\right) \leq 0 .
$$

Integrating over $(0, t)$, for all $t \in[0, T]$, we obtain

$$
\int_{0}^{A} \int_{0}^{1} u^{2}(t, a, x) d x d a \leq e^{C T} \int_{0}^{A} \int_{0}^{1} u^{2}(0, a, x) d x d a=e^{C T} \int_{0}^{A} \int_{0}^{1} y_{0}^{2}(a, x) d x d a .
$$

In particular,

$$
\int_{0}^{A} \int_{0}^{1} u^{2}(\tilde{T}, a, x) d x d a \leq e^{C T} \int_{0}^{A} \int_{0}^{1} y_{0}^{2}(a, x) d x d a
$$


Thus,

$$
\begin{aligned}
\left\|f_{\delta}\right\|_{L^{2}(Q)}^{2} & =\int_{\tilde{T}}^{T} \int_{0}^{A} \int_{0}^{1} h_{\delta}^{2} d x d a d t \leq C\left\|\tilde{y}_{0}\right\|_{L^{2}\left(Q_{A, 1}\right)}^{2} \\
& =C \int_{0}^{A} \int_{0}^{1} u^{2}(\tilde{T}, a, x) d x d a \leq C \int_{0}^{A} \int_{0}^{1} y_{0}^{2}(a, x) d x d a,
\end{aligned}
$$

for a strictly positive constant $C$. Hence, (4.29) follows.

As a consequence of the previous theorem, we obtain the null controllability property if the coefficient $k$ degenerates at 0 and at 1 at the same time.

Theorem 4.8. Assume Hypotheses 4.1 and 4.2. Then, given $T \in(0, A)$ and $y_{0} \in L^{2}\left(Q_{A, 1}\right)$, for every $\delta \in$ $(T, A)$, there exists a control $f_{\delta} \in L^{2}(Q)$ such that the solution $y_{\delta}$ of (1.1) satisfies

$$
y_{\delta}(T, a, x)=0 \quad \text { a.e. }(a, x) \in(\delta, A) \times(0,1) .
$$

Moreover, there exists $C=C(\delta)>0$ such that

$$
\left\|f_{\delta}\right\|_{L^{2}(Q)} \leq C\left\|y_{0}\right\|_{L^{2}\left(Q_{A, 1}\right)} .
$$

Proof. Fix $y_{0} \in L^{2}\left(Q_{A, 1}\right)$ and consider the two problems

$$
\left(P_{1}\right) \begin{cases}\frac{\partial y}{\partial t}+\frac{\partial y}{\partial a}-\left(k(x) y_{x}\right)_{x}+\mu(t, a, x) y=f(t, a, x) \chi_{\omega} & \text { in }(0, T) \times(0, A) \times(0, \bar{\beta}), \\ y(t, a, \bar{\beta})=y(t, a, 0)=0 & \text { on } Q_{T, A}, \\ y(0, a, x)=y_{0}(a, x) & \text { in }(0, A) \times(0, \bar{\beta}), \\ y(t, 0, x)=\int_{0}^{A} \beta(a, x) y(t, a, x) d a & \text { in }(0, T) \times(0, \bar{\beta}),\end{cases}
$$

and

$$
\left(P_{2}\right) \begin{cases}\frac{\partial y}{\partial t}+\frac{\partial y}{\partial a}-\left(k(x) y_{x}\right)_{x}+\mu(t, a, x) y=f(t, a, x) \chi_{\omega} & \text { in }(0, T) \times(0, A) \times(\bar{\alpha}, 1), \\ y(t, a, 1)=y(t, a, \bar{\alpha})=0 & \text { on } Q_{T, A}, \\ y(0, a, x)=y_{0}(a, x) & \text { in }(0, A) \times(\bar{\alpha}, 1), \\ y(t, 0, x)=\int_{0}^{A} \beta(a, x) y(t, a, x) d a & \text { in }(0, T) \times(\bar{\alpha}, 1),\end{cases}
$$

where $\bar{\alpha} \in(0, \alpha)$ and $\bar{\beta} \in(\beta, 1)$. Thus, by Theorem 4.7, there exist two controls $h_{1, \delta}$ and $h_{2, \delta}$ such that the solutions $u_{1, \delta}$ and $u_{2, \delta}$ of $\left(P_{1}\right)$ and $\left(P_{2}\right)$, associated to $h_{1, \delta}$ and $h_{2, \delta}$, respectively, satisfy

$$
u_{1, \delta}(T, a, x)=0 \quad \text { a.e. }(a, x) \in(\delta, A) \times(0, \bar{\beta}),
$$

and

$$
u_{2, \delta}(T, a, x)=0 \quad \text { a.e. }(a, x) \in(\delta, A) \times(\bar{\alpha}, 1) .
$$

Moreover, there exists $C>0$ such that

$$
\int_{0}^{T} \int_{0}^{A} \int_{0}^{\bar{\beta}} h_{1, \delta}^{2} d x d a d t \leq C\left\|y_{0}\right\|_{L^{2}\left(Q_{A, 1}\right)}^{2}
$$

and

$$
\int_{0}^{T} \int_{0}^{A} \int_{\bar{\alpha}}^{1} h_{2, \delta}^{2} d x d a d t \leq C\left\|y_{0}\right\|_{L^{2}\left(Q_{A, 1}\right)}^{2} .
$$

Denote with $u_{1}$ and $h_{1}$ (respectively $u_{2}$ and $h_{2}$ ) the trivial extensions of $u_{1, \delta}$ and $h_{1, \delta}$ (respectively $u_{2, \delta}$ and $h_{2, \delta}$ ) to $[\bar{\beta}, 1]$ (respectively $[0, \bar{\alpha}]$ ), so that all functions are defined in the interval $[0,1]$. Clearly, they depends always on $\delta$ and

$$
\left\|h_{i}\right\|_{L^{2}(Q)} \leq C\left\|y_{0}\right\|_{L^{2}\left(Q_{A, 1}\right)}, i=1,2 .
$$


Now, let $u_{3}$ be the solution of

$$
\begin{cases}\frac{\partial y}{\partial t}+\frac{\partial y}{\partial a}-\left(k(x) y_{x}\right)_{x}+\mu(t, a, x) y=0 & \text { in }(0, T) \times(0, A) \times(0,1), \\ y(t, a, 1)=y(t, a, 0)=0 & \text { on } Q_{T, A}, \\ y(0, a, x)=y_{0}(a, x) & \text { in }(0, A) \times(0,1), \\ y(t, 0, x)=\int_{0}^{A} \beta(a, x) y(t, a, x) d a & \text { in }(0, T) \times(0,1),\end{cases}
$$

and consider the three smooth cut off functions $\xi, \eta, \phi:[0,1] \rightarrow \mathbb{R}$ defined as

$$
\begin{aligned}
& \begin{cases}0 \leq \xi(x) \leq 1, & \text { for all } x \in[0,1], \\
\xi(x)=1, & x \in[0,(2 \alpha+\rho) / 3], \\
\xi(x)=0, & x \in[(\alpha+2 \rho) / 3,1],\end{cases} \\
& \begin{cases}0 \leq \eta(x) \leq 1, & \text { for all } x \in[0,1], \\
\eta(x)=0, & x \in[0,(2 \alpha+\rho) / 3], \\
\eta(x)=1, & x \in[(\alpha+2 \rho) / 3,1]\end{cases}
\end{aligned}
$$

and $\phi:=1-\xi-\eta$. Finally, take

$$
y(t, a, x)=\xi u_{1}+\eta u_{2}+F(t) \phi u_{3},
$$

where $F(t):=\frac{T-t}{T}$.

It is easy to verify that $y(t, a, 0)=y(t, a, 1)=0, y(0, a, x)=y_{0}(a, x)$ (since $\left.F(0)=1\right)$ and $y(t, 0, x)=$ $\int_{0}^{A} \beta(a, x) y(t, a, x) d a$. Moreover,

$$
y(T, a, x)=0 \quad \text { a.e. }(a, x) \in(\delta, A) \times(0,1)
$$

and $y$ satisfies the equation of (1.1) with

$$
\begin{aligned}
f_{\delta} & =\xi h_{1} \chi \omega+\eta h_{2} \chi \omega-\frac{1}{T} \phi u_{3}-F(t) k \phi^{\prime} u_{3, x}-F(t)\left(k \phi^{\prime} u_{3}\right)_{x} \\
& -k \xi^{\prime} u_{1, x}-\left(k \xi^{\prime} u_{1}\right)_{x}-k \eta^{\prime} u_{2, x}-\left(k \eta^{\prime} u_{2}\right)_{x} .
\end{aligned}
$$

Obviously, the support of $f_{\delta}$ is contained in $\omega$ and, since $k \in C^{1}(\bar{\omega})$, the terms $\left(k \phi^{\prime} u_{3}\right)_{x},\left(k \xi^{\prime} u_{1}\right)_{x}$ and $\left(k \eta^{\prime} u_{2}\right)_{x}$ are $L^{2}(0,1)$ (recall that $\phi^{\prime}(x)=\xi^{\prime}(x)=\eta^{\prime}(x)=0$ for all $\left.x \in(0,1) \backslash \omega\right)$; thus $f_{\delta} \in L^{2}(Q)$ as required. As in [19], estimate (4.33) follows by the definition of $f_{\delta}$, (4.36) and (2.2) for $u_{i}, i=1,2,3$.

Observe that the previous result can be proved also for the problem in non divergence form considered in [13].

\section{A Appendix}

\section{A.1 Proof of (2.2):}

Multiplying the equation of (1.1) by $y$ and integrating over $(0, A) \times(0,1)$, we obtain

$$
\begin{aligned}
& \frac{1}{2} \frac{d}{d t}\|y(t)\|_{L^{2}\left(Q_{A, 1}\right)}^{2}+\frac{1}{2} \int_{0}^{1} y^{2}(t, A, x) d x-\frac{1}{2} \int_{0}^{1} y^{2}(t, 0, x) d x+\int_{0}^{A} \int_{0}^{1} k y_{x}^{2} d x d a \\
& =-\int_{0}^{A} \int_{0}^{1} \mu y^{2} d x d a+\int_{0}^{A} \int_{\omega} f y d x d a .
\end{aligned}
$$


Hence, using the initial condition $y(t, 0, x)=\int_{0}^{A} \beta(a, x) y(t, a, x) d a$, the assumptions on $\beta$ and $\mu$ and the inequality of Jensen, one has

$$
\begin{aligned}
& \frac{1}{2} \frac{d}{d t}\|y(t)\|_{L^{2}\left(Q_{A, 1}\right)}^{2}+\frac{1}{2} \int_{0}^{1} y^{2}(t, A, x) d x+\int_{0}^{A} \int_{0}^{1} k y_{x}^{2} d x d a \\
& \leq \frac{C}{2} \int_{0}^{A} \int_{0}^{1} y^{2}(t, a, x) d x d a+\frac{1}{2} \int_{0}^{A} \int_{0}^{1} f^{2} d x d a,
\end{aligned}
$$

where $C$ is a positive constant. Since $\int_{0}^{1} y^{2}(t, A, x) d x$ and $\int_{0}^{A} \int_{0}^{1} k y_{x}^{2} d x d a$ are positive, we deduce

$$
\frac{d}{d t}\|y(t)\|_{L^{2}\left(Q_{A, 1}\right)}^{2} \leq C\|y(t)\|_{L^{2}\left(Q_{A, 1}\right)}^{2}+\|f(t)\|_{L^{2}\left(Q_{A, 1}\right)}^{2} .
$$

Setting $F(t):=\|y(t)\|_{L^{2}\left(Q_{A, 1}\right)}^{2}$ and multiplying the previous inequality by $e^{-C t}$, one has

$$
\frac{d}{d t}\left(e^{-C t} F(t)\right) \leq e^{-C t}\|f(t)\|_{L^{2}\left(Q_{A, 1}\right.}^{2} .
$$

Integrating (A.2) over $(0, t)$, for all $t \in[0, T]$ it follows

$$
e^{-C t} F(t) \leq F(0)+\int_{0}^{t} e^{-C \tau}\|f(\tau)\|_{L^{2}\left(Q_{A, 1}\right)}^{2} d \tau
$$

Hence, for all $t \in[0, T]$,

$$
F(t) \leq e^{C T}\left(F(0)+\int_{0}^{T}\|f(\tau)\|_{L^{2}\left(Q_{A, 1}\right)}^{2} d \tau\right)
$$

and

$$
\sup _{t \in[0, T]}\|y(t)\|_{L^{2}\left(Q_{A, 1}\right)}^{2} \leq C\left(\left\|y_{0}\right\|_{L^{2}\left(Q_{A, 1}\right)}^{2}+\|f\|_{L^{2}(Q)}^{2} d \tau\right) .
$$

Therefore, by (A.1), it follows

$$
\frac{1}{2} \frac{d}{d t}\|y(t)\|_{L^{2}\left(Q_{A, 1}\right)}^{2}+\int_{0}^{A} \int_{0}^{1} k y_{x}^{2} d x d a \leq \frac{C}{2} \int_{0}^{A} \int_{0}^{1} y^{2}(t, a, x) d x d a+\frac{1}{2} \int_{0}^{A} \int_{0}^{1} f^{2} d x d a .
$$

Integrating over $(0, T)$, we have

$$
\begin{aligned}
\frac{1}{2}\|y(T)\|_{L^{2}\left(Q_{A, 1}\right)}^{2}+\int_{0}^{T} \int_{0}^{A} \int_{0}^{1} k y_{x}^{2} d x d a d t & \leq \frac{1}{2}\left\|y_{0}\right\|_{L^{2}\left(Q_{A, 1}\right)}^{2}+\frac{C}{2} \int_{0}^{T} \int_{0}^{A} \int_{0}^{1} y^{2}(t, a, x) d x d a d t \\
& +\frac{1}{2} \int_{0}^{T} \int_{0}^{T} \int_{0}^{1} f^{2} d x d a d t .
\end{aligned}
$$

Hence, by (A.3),

$$
\begin{aligned}
\int_{0}^{T} \int_{0}^{A}\left\|\sqrt{k} y_{X}\right\|_{L^{2}(0,1)}^{2} d a d t & \leq\left\|y_{0}\right\|_{L^{2}\left(Q_{A, 1}\right)}^{2}+C \int_{0}^{T}\|y(t)\|_{L^{2}\left(Q_{A, 1}\right)}^{2} d t+\|f\|_{L^{2}(Q)}^{2} \\
& \leq C\left(\left\|y_{0}\right\|_{L^{2}\left(Q_{A, 1}\right)}^{2}+\|f\|_{L^{2}(Q)}^{2} d \tau\right)
\end{aligned}
$$

and (2.2) follows by (A.3) and (A.4). 


\section{A.2 Proof of Proposition 3.2:}

We consider case (i), Hypothesis (HP1). Fix $\beta \in(\theta, 1)$ arbitrarily for the moment. Since $w(1)=0$, we have

$$
\int_{0}^{1} \frac{k(x)}{(1-x)^{2}} w^{2}(x) d x=\int_{0}^{1} \frac{k(x)}{(1-x)^{2}}\left(\int_{x}^{1}(1-y)^{\beta / 2} w^{\prime}(y)(1-y)^{-\beta / 2} d y\right)^{2} d x .
$$

This implies

$$
\int_{0}^{1} \frac{k(x)}{(1-x)^{2}} w^{2}(x) d x \leq \int_{0}^{1} \frac{k(x)}{(1-x)^{2}}\left(\int_{x}^{1}(1-y)^{\beta}\left|w^{\prime}(y)\right|^{2} d y \int_{x}^{1}(1-y)^{-\beta} d y\right) d x .
$$

Hence, we have

$$
\int_{0}^{1} \frac{k(x)}{(1-x)^{2}} w^{2}(x) d x \leq \frac{1}{1-\beta} \int_{0}^{1} \frac{k(x)}{(1-x)^{1+\beta}}\left(\int_{x}^{1}(1-y)^{\beta}\left|w^{\prime}(y)\right|^{2} d y\right) d x .
$$

By the Theorem of Fubini, it follows

$$
\int_{0}^{1} \frac{k(x)}{(1-x)^{2}} w^{2}(x) d x \leq \frac{1}{1-\beta} \int_{0}^{1}(1-y)^{\beta}\left|w^{\prime}(y)\right|^{2}\left(\int_{0}^{y} \frac{k(x)}{(1-x)^{1+\beta}} d x\right) d y .
$$

Now, divide the right hand side of (A.5) into three parts, i.e.

$$
\int_{0}^{1}(1-y)^{\beta}\left|w^{\prime}(y)\right|^{2}\left(\int_{0}^{y} \frac{k(x)}{(1-x)^{1+\beta}} d x\right) d y=L_{\epsilon}+M_{\epsilon}+N_{\epsilon},
$$

where

$$
\begin{aligned}
L_{\epsilon} & =\int_{0}^{1-\epsilon}(1-y)^{\beta}\left|w^{\prime}(y)\right|^{2}\left(\int_{0}^{y} \frac{k(x)}{(1-x)^{1+\beta}} d x\right) d y, \\
M_{\epsilon} & =\int_{1-\epsilon}^{1}(1-y)^{\beta}\left|w^{\prime}(y)\right|^{2}\left(\int_{0}^{1-\epsilon} \frac{k(x)}{(1-x)^{1+\beta}} d x\right) d y,
\end{aligned}
$$

and

$$
N_{\epsilon}=\int_{1-\epsilon}^{1}(1-y)^{\beta}\left|w^{\prime}(y)\right|^{2}\left(\int_{1-\epsilon}^{y} \frac{k(x)}{(1-x)^{1+\beta}} d x\right) d y .
$$

Thanks to our hypothesis, there exists $\epsilon>0$ such that the function

$$
x \longrightarrow \frac{k(x)}{(1-x)^{\theta}} \text { is nondecreasing on }[1-\epsilon, 1) ;
$$

thus, for $N_{\epsilon}$, we have

$$
\begin{aligned}
N_{\epsilon} & =\int_{1-\epsilon}^{1}(1-y)^{\beta}\left|w^{\prime}(y)\right|^{2}\left(\int_{1-\epsilon}^{y} \frac{k(x)}{(1-x)^{1+\beta}} d x\right) d y \\
& \leq \int_{1-\epsilon}^{1}(1-y)^{\beta-\theta}\left|w^{\prime}(y)\right|^{2} k(y)\left(\int_{1-\epsilon}^{y}(1-x)^{\theta-\beta-1} d x\right) d y \leq \frac{1}{(\beta-\theta)} \int_{1-\epsilon}^{1} k(y)\left|w^{\prime}(y)\right|^{2} d y .
\end{aligned}
$$


For $M_{\epsilon}$, we have

$$
\begin{aligned}
M_{\epsilon} & \leq \sup _{[0,1-\epsilon]} k \int_{1-\epsilon}^{1}(1-y)^{\beta} \frac{k(y)}{k(y)}\left|w^{\prime}(y)\right|^{2}\left(\int_{0}^{1-\epsilon}(1-x)^{-(1+\beta)} d x\right) d y \\
& \leq \frac{\epsilon^{\theta-\beta}}{\beta k(1-\epsilon)} \sup _{[0,1-\epsilon]} k \int_{1-\epsilon}^{1}(1-y)^{\beta-\theta} k(y)\left|w^{\prime}(y)\right|^{2} \leq C \int_{1-\epsilon}^{1} k(x)\left|w^{\prime}(x)\right|^{2} d x .
\end{aligned}
$$

Proceeding in a similar way, we obtain

$$
L_{\epsilon} \leq C \int_{0}^{1-\epsilon} k(x)\left|w^{\prime}(x)\right|^{2} d x
$$

Using (A.6), (A.7) and (A.8) in (A.5), we obtain

$$
\int_{0}^{1} \frac{k(x)}{(1-x)^{2}} w^{2}(x) d x \leq C \int_{0}^{1} k(x)\left|w^{\prime}(x)\right|^{2} d x
$$

where the constant $C$ depends on $a, \epsilon, \theta$ and $\beta$. If one assumes that Hypothesis (HP1)' holds, that is

$$
x \longrightarrow \frac{k(x)}{(1-x)^{\theta}} \text { is nondecreasing on }[0,1),
$$

then, one can take $\epsilon=1$ in the above computations, so that $L_{\epsilon}=M_{\epsilon}=0$. Using then (A.6) in (A.5), with $\epsilon=1$, one obtains

$$
\int_{0}^{1} \frac{k(x)}{(1-x)^{2}} w^{2}(x) d x \leq \frac{1}{(1-\beta)(\beta-\theta)} \int_{0}^{1} k(x)\left|w^{\prime}(x)\right|^{2} d x .
$$

We then remark that this last estimate is optimal for $\beta=\frac{\theta+1}{2}$, which gives the desired result.

We now consider case (ii), Hypothesis (HP2). Fix $\beta \in(1, \theta)$ arbitrarily for the moment. As before

$$
\int_{0}^{1} \frac{k(x)}{(1-x)^{2}} w^{2}(x) d x=\int_{0}^{1} \frac{k(x)}{(1-x)^{2}}\left(\int_{0}^{x}(1-y)^{\beta / 2} w^{\prime}(y)(1-y)^{-\beta / 2} d y\right)^{2} d x
$$

so that

$$
\int_{0}^{1} \frac{k(x)}{(1-x)^{2}} w^{2}(x) d x \leq \int_{0}^{1} \frac{k(x)}{(1-x)^{2}}\left(\int_{0}^{x}(1-y)^{\beta}\left|w^{\prime}(y)\right|^{2} d y \int_{0}^{x}(1-y)^{-\beta} d y\right) d x .
$$

It follows that

$$
\int_{0}^{1} \frac{k(x)}{(1-x)^{2}} w^{2}(x) d x \leq \frac{1}{\beta-1} \int_{0}^{1} \frac{k(x)}{(1-x)^{1+\beta}}\left(\int_{0}^{x}(1-y)^{\beta}\left|w^{\prime}(y)\right|^{2} d y\right) d x .
$$

Applying the Theorem of Fubini, we have

$$
\int_{0}^{1} \frac{k(x)}{(1-x)^{2}} w^{2}(x) d x \leq \frac{1}{\beta-1} \int_{0}^{1}(1-y)^{\beta}\left|w^{\prime}(y)\right|^{2}\left(\int_{y}^{1} \frac{k(x)}{(1-x)^{1+\beta}} d x\right) d y .
$$

As before, we rewrite

$$
\int_{0}^{1}(1-y)^{\beta}\left|w^{\prime}(y)\right|^{2}\left(\int_{y}^{1} \frac{k(x)}{(1-x)^{1+\beta}} d x\right) d y=I_{\epsilon}+J_{\epsilon}+K_{\epsilon},
$$


where

$$
\begin{aligned}
I_{\epsilon} & =\int_{0}^{1-\epsilon}(1-y)^{\beta}\left|w^{\prime}(y)\right|^{2}\left(\int_{y}^{1-\epsilon} \frac{k(x)}{(1-x)^{1+\beta}} d x\right) d y, \\
J_{\epsilon} & =\int_{0}^{1-\epsilon}(1-y)^{\beta}\left|w^{\prime}(y)\right|^{2}\left(\int_{1-\epsilon}^{1} \frac{k(x)}{(1-x)^{1+\beta}} d x\right) d y
\end{aligned}
$$

and

$$
K_{\epsilon}=\int_{1-\epsilon}^{1}(1-y)^{\beta}\left|w^{\prime}(y)\right|^{2}\left(\int_{y}^{1} \frac{k(x)}{(1-x)^{1+\beta}} d x\right) d y .
$$

Thanks to our hypothesis, there exists $\epsilon>0$ such that the function

$$
x \longrightarrow \frac{k(x)}{(1-x)^{\theta}} \text { is nonincreasing on }[1-\epsilon, 1),
$$

thus $K_{\epsilon}$ can be estimated in the following way:

$$
\begin{aligned}
K_{\epsilon} & =\int_{1-\epsilon}^{1}(1-y)^{\beta}\left|w^{\prime}(y)\right|^{2}\left(\int_{y}^{1} \frac{k(x)}{(1-x)^{1+\beta}} d x\right) d y \\
& \leq \int_{1-\epsilon}^{1}(1-y)^{\beta}\left|w^{\prime}(y)\right|^{2} \frac{k(y)}{(1-y)^{\theta}} \int_{y}^{1}(1-x)^{\theta-\beta-1} d x \leq \frac{1}{(\theta-\beta)} \int_{1-\epsilon}^{1} k(x)\left|w^{\prime}(x)\right|^{2} d x .
\end{aligned}
$$

For $J_{\epsilon}$, we can proceed in a similar way, obtaining

$$
J_{\epsilon}=\int_{0}^{1-\epsilon}(1-y)^{\beta}\left|w^{\prime}(y)\right|^{2}\left(\int_{1-\epsilon}^{1} \frac{k(x)}{(1-x)^{1+\beta}} d x\right) d y \leq \epsilon^{-\beta} \frac{k(1-\epsilon)}{\inf _{[0,1-\epsilon]} k} \int_{0}^{1-\epsilon} k(y)\left|w^{\prime}(y)\right|^{2} d y .
$$

For $I_{\epsilon}$, we have

$$
\begin{aligned}
I_{\epsilon} & =\int_{0}^{1-\epsilon}(1-y)^{\beta}\left|w^{\prime}(y)\right|^{2} \frac{k(y)}{k(y)}\left(\int_{y}^{1-\epsilon} \frac{k(x)}{(1-x)^{1+\beta}} d x\right) d y \\
& \leq \frac{\sup _{[0,1-\epsilon]} k}{\beta \inf _{[0,1-\epsilon]} k} \int_{0}^{1-\epsilon} k(y)\left|w^{\prime}(y)\right|^{2}(1-y)^{\beta} \epsilon^{-\beta} d y \leq C \int_{0}^{1-\epsilon} k(x)\left|w^{\prime}(x)\right|^{2} d x .
\end{aligned}
$$

Using (A.11), (A.12) and (A.13) in (A.10), we obtain

$$
\int_{0}^{1} \frac{k(x)}{(1-x)^{2}} w^{2}(x) d x \leq C \int_{0}^{1} k(x)\left|w^{\prime}(x)\right|^{2} d x,
$$

where the constant $C$ depends on $a, \epsilon, \theta$ and $\beta$. If one assumes that hypothesis (HP2)' holds, that is

$$
x \rightarrow \frac{k(x)}{(1-x)^{\theta}} \text { is noninreasing on }[0,1),
$$

then, one can take $\epsilon=1$ in the above computations, so that $J_{\epsilon}=I_{\epsilon}=0$. Using then (2.1) in (A.10), with $\epsilon=1$, one obtains

$$
\int_{0}^{1} \frac{k(x)}{(1-x)^{2}} w^{2}(x) d x \leq \frac{1}{(\beta-1)(\theta-\beta)} \int_{0}^{1} k(x)\left|w^{\prime}(x)\right|^{2} d x .
$$

We then remark that this last estimate is optimal for $\beta=\frac{\theta+1}{2}$, which gives the desired result. 


\section{A.3 Proof of Proposition 4.2:}

Let us consider a smooth function $\xi:[0,1] \rightarrow \mathbb{R}$ such that

$$
\begin{cases}0 \leq \xi(x) \leq 1, & \text { for all } x \in[0,1], \\ \xi(x)=1, & x \in \omega^{\prime}, \\ \xi(x)=0, & x \in(0,1) \backslash \omega .\end{cases}
$$

Then, integrating by parts one has

$$
\begin{aligned}
0= & \int_{0}^{T} \frac{d}{d t}\left(\int_{0}^{A} \int_{0}^{1}\left(\xi e^{s \psi}\right)^{2} v^{2} d x d a\right) d t \\
& =\int_{Q} 2 s \psi_{t}\left(\xi e^{s \psi}\right)^{2} v^{2}+2\left(\xi e^{s \psi}\right)^{2} v\left(-v_{a}-\left(k v_{x}\right)_{x}+\mu v+f\right) d x d a d t \\
& =2 s \int_{Q} \psi_{t}\left(\xi e^{s \psi}\right)^{2} v^{2} d x d a d t+2 s \int_{Q} \psi_{a}\left(\xi e^{s \psi}\right)^{2} v^{2} d x d a d t+2 \int_{Q}\left(\xi^{2} e^{2 s \psi}\right)_{x} k v v_{x} d x d a d t \\
& +2 \int_{Q}\left(\xi^{2} e^{2 s \psi} k\right) v_{x}^{2} d x d a d t+2 \int_{Q} \xi^{2} e^{2 s \psi} \mu v^{2} d x d a d t+2 \int_{Q} \xi^{2} e^{2 s \psi} f v d x d a d t .
\end{aligned}
$$

Hence, using Young's inequality

$$
\begin{aligned}
2 \int_{Q} \xi^{2} e^{2 s \psi} k v_{x}^{2} d x d a d t & =-2 s \int_{Q} \psi_{t}\left(\xi e^{s \psi}\right)^{2} v^{2} d x d a d t-2 s \int_{Q} \psi_{a}\left(\xi e^{s \psi}\right)^{2} v^{2} d x d a d t \\
& -2 \int_{Q}\left(\xi^{2} e^{2 s \psi}\right)_{x} k v v_{x} d x d a d t-2 \int_{Q} \xi^{2} e^{2 s \psi} \mu v^{2} d x d a d t \\
& -2 \int_{Q} \xi^{2} e^{2 s \psi} f v d x d a d t \\
& \leq-2 s \int_{Q} \psi_{t}\left(\xi e^{s \psi}\right)^{2} v^{2} d x d a d t-2 s \int_{Q} \psi_{a}\left(\xi e^{s \psi}\right)^{2} v^{2} d x d a d t \\
& +\int_{Q}\left(\sqrt{k} \frac{\left(\xi^{2} e^{2 s \varphi}\right)_{x}}{\xi e^{s \varphi}} v\right)^{2} d x d a d t+\int_{Q} \xi^{2} e^{2 s \varphi} k v_{x}^{2} d x d a d t . \\
& +\left(2\|\mu\|_{L^{\infty}(Q)}+1\right) \int_{Q} \xi^{2} v^{2} d x d a d t+\int_{Q} \xi^{2} e^{2 s \psi} f^{2} d x d a d t .
\end{aligned}
$$

Thus,

$$
\begin{aligned}
& \inf _{\omega^{\prime}}\{k\} \int_{0}^{T} \int_{0}^{A} \int_{\omega^{\prime}} e^{2 s \psi} v_{x}^{2} d x d a d t \\
& \left.\leq \sup _{\omega \times(0, T)}\left\{\left|4 k\left(\xi e^{s \psi}\right)_{x}^{2}-2 s\left(\psi_{t}+\psi_{a}\right)\left(\xi e^{s \psi}\right)^{2}\right|\right\}+2\|\mu\|_{L^{\infty}(Q)}+1\right) \int_{0}^{T} \int_{0}^{A} \int_{\omega} v^{2} d x d a d t \\
& +\int_{Q} f^{2} e^{2 s \psi} d x d a d t .
\end{aligned}
$$

Acknowledgments: The author is a member of the Gruppo Nazionale per l'Analisi Matematica, la Probabilità e le loro Applicazioni (GNAMPA) of the Istituto Nazionale di Alta Matematica (INdAM) and she is supported 
by the FFABR "Fondo per il finanziamento delle attività base di ricerca” 2017, by the INdAM- GNAMPA Project 2019 "Controllabilità di PDE in modelli fisici e in scienze della vita" and by PRIN 2017-2019 Qualitative and quantitative aspects of nonlinear PDEs.

\section{References}

[1] B. Ainseba, Y. Echarroudi, L. Maniar Null controllability of population dynamics with degenerate diffusion, Differential Integral Equations (2013), 1397-1410.

[2] F. Alabau-Boussouira, P. Cannarsa, G Fragnelli, Carleman estimates for degenerate parabolic operators with applications to null controllability, J. Evol. Equ. 6 (2006), 161-204.

[3] S. Aniţa, Analysis and control of age-dependent population dynamics, Mathematical Modelling: Theory and Applications 11 (2000), Kluwer Academic Publishers, Dordrecht.

[4] V. Barbu, M. Iannelli, M. Martcheva, On the controllability of the Lotka-McKendrick model of population dynamics, J. Math. Anal. Appl. 253 (2001), 142-165.

[5] R. Borges, A. Calsina, S. Cuadrado, O. Diekmann, Delay equation formulation of a cyclin-structured cell population model, J. Evol. Equ. 14 (2014), 841-862.

[6] H. Brezis, Functional Analysis, Sobolev Spaces and Partial Differential Equations, Springer Science+Business Media, LLC 2011.

[7] P. Cannarsa, G. Fragnelli, D. Rocchetti, Controllability results for a class of one-dimensional degenerate parabolic problems in nondivergence form, J. Evol. Equ. 8 (2008), 583-616.

[8] P. Cannarsa, P. Martinez, J. Vancostenoble, Persistent regional controllability for a class of degenerate parabolic equations, Commun. Pure Appl. Anal. 3 (2004), 607-635.

[9] O. Diekmann, Ph. Getto, Boundedness, global existence and continuous dependence for nonlinear dynamical systems describing physiologically structured populations, J. Differential Equations 215 (2005), 268-319.

[10] Y. Echarroudi, L. Maniar, Null controllability of a model in population dynamics, Electron. J. Differential Equations 2014 (2014), 1-20.

[11] K.J. Engel, R. Nagel, One-Parameter Semigroups for Linear Evolution Equations, Springer-Verlag, New York, Berlin, Heidelberg, 1999.

[12] E. Fernández-Cara, S. Guerrero, Global Carleman inequalities for parabolic systems and applications to controllability, SIAM J. Control Optim. 45, 1395-1446.s

[13] G. Fragnelli, Carleman estimates and null controllability for a degenerate population model, Journal de Mathématiques Pures et Appliqués 115 (2018), 74-126.

[14] G. Fragnelli, P. Martinez, J. Vancostenoble, Qualitative properties of a population dynamics system describing pregnancy, Math. Models Methods Appl. Sci. 15 (2005), 507-554.

[15] G. Fragnelli, D. Mugnai, Carleman estimates and observability inequalities for parabolic equations with interior degeneracy, Advances in Nonlinear Analysis 2 (2013), 339-378.

[16] G. Fragnelli, D. Mugnai, Carleman estimates, observability inequalities and null controllability for interior degenerate non smooth parabolic equations, Mem. Amer. Math. Soc. 242 (2016), v+84 pp. Corrigendum, to appear.

[17] G. Fragnelli, D. Mugnai, Carleman estimates for singular parabolic equations with interior degeneracy and non smooth coefficients, Adv. Nonlinear Anal. 6 (2017), 61-84.

[18] G. Fragnelli, D. Mugnai, Controllability of strongly degenerate parabolic problems with strongly singular potentials, Electron. J. Qual. Theory Differ. Equ. 50 (2018), 1-11.

[19] G. Fragnelli, D. Mugnai, Controllability of degenerate and singular parabolic problems: the double strong case with Neumann boundary conditions, Opuscula Math. 39 (2019), 207-225.

[20] G. Fragnelli, L. Tonetto, A population equation with diffusion, J. Math. Anal. Appl. 289 (2004), 90-99.

[21] Y. He, B. Ainseba, Exact null controllability of the Lobesia botrana model with diffusion, J. Math. Anal. Appl. 409 (2014), 530-543.

[22] J.L. Lions, E. Magenes, Non-homogeneous boundary value problems and applications. Vol. I. Translated from the French by P. Kenneth. Die Grundlehren der mathematischen Wissenschaften, Band 181. Springer-Verlag, New York-Heidelberg (1972).

[23] D. Maity, M. Tucsnak and E. Zuazua, Controllability and positivity constraints in population dynamics with age structuring and diffusion, Journal de Mathématiques Pures et Appliqués. In press, 10.1016/j.matpur.2018.12.006.

[24] S. S. Win, R. Muhamad, Z. A. M. Ahmad, N. A. Adam, Population Fluctuations of Brown Plant Hopper Nilaparvata lugens Stal. and White Backed Plant Hopper Sogatella furcifera Horvath on Rica, Jounal of Entomology 8 (2011), 183-190.

[25] B. Zhong, C. Lv and W. Qin, Effect of Temperature on the Population Growth of Tirathaba rufivena (Lepidoptera: Pyralidae) on Areca catechu (Arecaceae), Florida Entomologist 100 (2017), 578-582. 